\title{
AAV-mediated follistatin gene therapy improves functional outcomes in the TIC-DUX4 mouse model of FSHD
}

Carlee R. Giesige, ${ }^{1,2}$ Lindsay M. Wallace, ${ }^{2}$ Kristin N. Heller, ${ }^{2}$ Jocelyn O. Eidahl, ${ }^{2}$ Nizar Y. Saad, ${ }^{2}$ Allison M. Fowler, ${ }^{2}$ Nettie K. Pyne, ${ }^{2}$ Mustafa Al-Kharsan, ${ }^{2}$ Afrooz Rashnonejad, ${ }^{2}$ Cholamhossein Amini Chermahini, ${ }^{2}$ Jacqueline S. Domire, ${ }^{2}$ Diana Mukweyi, ${ }^{2}$ Sara E. Garwick-Coppens, ${ }^{2}$ Susan M. Guckes, ${ }^{2}$ K. John McLaughlin, ${ }^{2,3}$ Kathrin Meyer, ${ }^{2}$ Louise R. Rodino-Klapac, ${ }^{2,3}$ and Scott Q. Harper ${ }^{1,2,3}$

'Biomedical Sciences Graduate Program, The Ohio State University, Columbus, Ohio, USA. ${ }^{2}$ Center for Gene Therapy, The Research Institute at Nationwide Children's Hospital, Columbus, Ohio, USA. ${ }^{3}$ Department of Pediatrics, The Ohio State University, Columbus, Ohio, USA.

Facioscapulohumeral muscular dystrophy (FSHD) is an autosomal dominant or digenic disorder linked to derepression of the toxic DUX4 gene in muscle. There is currently no pharmacological treatment. The emergence of DUX4 enabled development of cell and animal models that could be used for basic and translational research. Since DUX4 is toxic, animal model development has been challenging, but progress has been made, revealing that tight regulation of DUX4 expression is critical for creating viable animals that develop myopathy. Here, we report such a model - the tamoxifen-inducible FSHD mouse model called TIC-DUX4. Uninduced animals are viable, born in Mendelian ratios, and overtly indistinguishable from WT animals. Induced animals display significant DUX4-dependent myopathic phenotypes at the molecular, histological, and functional levels. To demonstrate the utility of TIC-DUX4 mice for therapeutic development, we tested a gene therapy approach aimed at improving muscle strength in DUX4-expressing muscles using adeno-associated virus serotype 1.Follistatin (AAV1.Follistatin), a natural myostatin antagonist. This strategy was not designed to modulate DUX4 but could offer a mechanism to improve muscle weakness caused by DUX4-induced damage. AAV1.Follistatin significantly increased TIC-DUX4 muscle mass and strength even in the presence of DUX4 expression, suggesting that myostatin inhibition may be a promising approach to treat FSHD-associated weakness. We conclude that TICDUX4 mice are a relevant model to study DUX4 toxicity and, importantly, are useful in therapeutic development studies for FSHD.

Conflict of interest: The authors have declared that no conflict of interest exists.

License: Copyright 2018, American Society for Clinical Investigation.

Submitted: July 27, 2018

Accepted: October 10, 2018

Published: November 15, 2018

Reference information: JCI Insight. 2018;3(22):e123538. https://doi.org/10.1172/jici. insight.123538.

\section{Introduction}

Facioscapulohumeral muscular dystrophy (FSHD) is a complex genetic disorder affecting up to 870,000 people worldwide (1-4). FSHD typically arises in young adulthood, and most patients develop clinical features in the second or third decade of life (1). Classical FSHD presentation includes progressive weakness in muscles of the face, shoulder-girdle, upper arms, and lower legs, although there may be wide variability in muscles affected, clinical severity, rate of progression, and age-at-onset (5). Patients also often describe myopathic pain (1). There is a critical need for FSHD therapies, but to our knowledge, no effective pharmacological treatments currently exist that slow progression or improve muscle strength.

There are 2 clinically indistinguishable forms of FSHD, called FSHD1 and FSHD2. The majority of cases ( 95\%) are classified as FSHD1, while the remainder are classified as FSHD2 or show typical FSHD presentation but are not yet genetically characterized. Stated simply, both forms of FSHD are caused by derepression of the toxic DUX4 gene (6-9). The DUX4 open-reading frame is encoded within D4Z4 repeats located on the human chromosome $4 \mathrm{q}$ subtelomere. Humans may have different copy numbers of D4Z4 repeats on both $4 \mathrm{q}$ alleles. D4Z4 arrays larger than 10 in number are typically embedded in heterochromatin, and as a result, the DUX4 gene located in each repeat is suppressed (10). FSHD1 is caused by a congenital reduction in the number of D4Z4 repeats on 1 allele (1-10 D4Z4 copies), which in turn disrupts the 
epigenetic silencing of the region. FSHD2 results from mutations in chromatin modifier genes ( $S M C H D 1$, $D N M T 3 B)$ that also lead to epigenetic derepression of $\mathrm{D} 4 \mathrm{Z} 4$ repeats $(8,10-17)$. In both instances, the $D U X 4$ gene can be transcribed into DUX4 mRNA. However, reduced epigenetic silencing of DUX4 is not sufficient to give rise to FSHD because the individual repeats lack polyA signals to stabilize DUX4 transcript (10). Inheritance of a specific chromosomal background - called 4qA, which contains an additional untranslated exon harboring a noncanonical polyA signal (called the pLAM region), located adjacent to the last repeat - is required to cause FSHD (18). The pLAM region contributes a polyA signal to the last DUX4 copy. Thus, if DUX4 transcription occurs on the 4qA haplotype, the full-length $D U X 4$ transcript located nearest the telomere is stabilized and translated into DUX4 protein, which is toxic to muscle (10).

The emergence of DUX4 derepression as an underlying pathogenic event in FSHD was momentous for the field because it allowed for development of cell and animal models, which are essential tools for therapeutic studies. Numerous important models created during the past decade revealed the toxic nature of DUX4 and demonstrated that tight temporal and cell-type regulation was required to produce viable animals (19-26). The earliest such model (iDUX[2.7]) was particularly instructive, as it demonstrated that even barely detectable amounts of leaky $D U X 4$ expression might be sufficient to cause embryonic lethality or produce phenotypes not typically associated with a muscular dystrophy, such as alopecia, runting, and hyperactivity (25). With these lessons in mind, several years ago, we set out to generate an FSHD-like mouse model that (a) contained a single copy of a human DUX4 gene at a defined, epigenetically open locus and (b) employed a Cre-recombinase inducible system to regulate $D U X 4$ expression as tightly as possible.

Here, we report the generation and extensive characterization of such a model: the tamoxifen (Tam) inducible Cre-DUX4 (TIC-DUX4) mouse line. In line with our goals, this system permits temporal and spatial DUX4 expression control, and TIC-DUX4 animals treated with Tam develop numerous DUX4-driven myopathic phenotypes at the molecular, histopathological, and functional levels. Phenotypes are Tam- and DUX4-dose responsive. Here, we extensively characterized this DUX4-induced phenotype in several muscle groups and untreated control mice at a broad age range ( 8 weeks-1.5 years). We developed a Tam dosing strategy that permitted long-term study of low DUX4 expression and more slowly progressive disease, which is amenable to testing outcome measures in FSHD therapeutic strategies in vivo. Toward this end, we tested an adeno-associated virus serotype 1.Follistatin (AAV1.Follistatin) gene therapy approach that improved whole muscle strength in TIC-DUX4 mice even in the presence of DUX4. Our data provide proof-of-principle supporting the use of this myostatin-inhibition therapy to treat muscle weakness caused by $D U X 4$ expression, even in the absence of DUX4-modulating therapies, and validate the utility of the TIC-DUX4 mouse model as a suitable tool to test such therapies.

\section{Results}

Generation of viable TIC-DUX4 mice. Our goal was to generate an FSHD-like mouse model containing a single copy of an inducible DUX4 transgene at a defined locus. To accomplish this, we designed a targeting construct and inserted a single DUX4 ORF (exon 1), including the downstream 3' UTR and polyA (pLAM) regions (introns 1 and 2 and exons 2 and 3), into the ROSA26 locus using traditional homologous recombination technology (Figure 1A) (27). To facilitate DUX4 protein detection, we added an in-frame V5 epitope tag to the DUX4 COOH-terminus. To prevent the endogenous ROSA26 promoter from driving unregulated $D U X 4$ expression, our targeting construct also included a Neomycin-resistance (Neo-resistance) cassette flanked by LoxP sites upstream of the DUX4 ORF (Figure 1A). We were uncertain about the efficiency of the noncanonical endogenous DUX4 poly-adenylation signal (PAS) (ATTAAA) located in $D U X 4$ exon 3, so we included a second PAS sequence (the canonical bovine growth hormone [BGH] PAS - AATAAA) downstream of the DUX4 3' UTR.

Following transfection of the targeting construct into mouse embryonic stem (ES) cells, we harvested DNA from Neo-resistant clones and performed Southern blot analysis on 129S6/SvEvTac ES cell genomic DNA digested with EcoRV. We confirmed insertion of the targeting construct in 16 lines, including the representative clone shown in Figure 1B, and we then used 1 properly targeted clone to generate mice (ROSA26DUX4 line). Heterozygous animals were backcrossed onto C57BL/6 and are currently at 11 generations. ROSA26-DUX4 mouse genotypes were confirmed via PCR (Supplemental Figure 1A, left; supplemental material available online with this article; https://doi.org/10.1172/jci.insight.123538DS1). Single transgenic mice were healthy, fertile, and indistinguishable from littermates. When Cre recombinase was introduced 
A
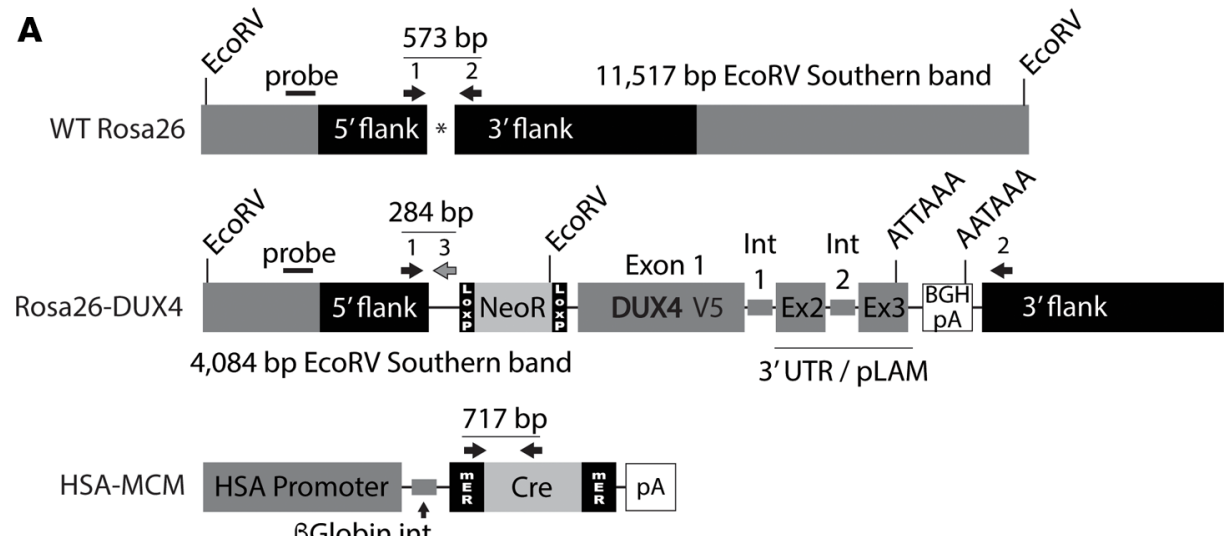

BGlobin int
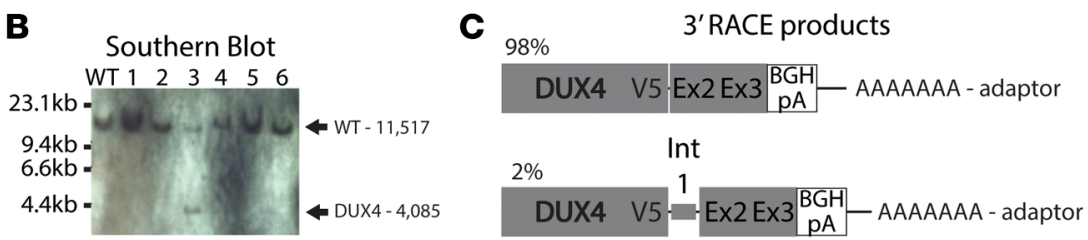

D DUX4 Western Blot

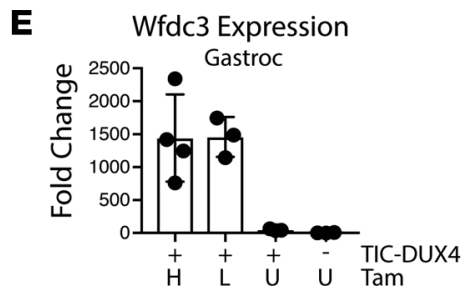

Figure 1. Generation of the ROSA26-DUX4 knock-in mouse and DUX4 expression validation in TIC-DUX4 mice. (A) Top, WT Rosa26 locus. Asterisk marks pRosa26-DUX4 insertion site. Black rectangles represent Rosa26 5' and 3' flanks. Gray rectangles depict Rosa26 genomic DNA sequences not included in the targeting construct. Middle, targeting construct inserted into Rosa26. The 5' end contains a LoxP-flanked (floxed) neomycin resistance gene (NeoR) driven by the PGK promoter (not shown). Adjacent to the NeoR cassette is 1 DUX4 ORF (exon 1) fused to a V5 epitope sequence, followed by introns (Int1, Int2) and 3' UTR exons (Ex2 and Ex3) from the last repeat of DUX4 (labeled 3' UTR/pLAM). Exon 3 derives from the chrom $4 q$ pLAM region and contains the noncanonical 5'-ATTAAA- $3^{\prime}$ polyA signal utilized by the last DUX4 copy in FSHD patients. When present, the floxed NeoR cassette inhibits DUX4 expression from the Rosa26 promoter. A bovine growth hormone polyA signal (BCH pA; 5'-AATAAA-3') was included next to DUX4/pLAM. The HSA-mER-Cre-mER (HSAMCM) mouse was previously published. HSA, human skeletal actin promoter with $\beta$-globin intron ( $\beta C$ lobin-int); mER, mutated estrogen receptor. Arrows indicate PCR primers used to detect WT Rosa26 and the knocked-in transgene in mouse genomic DNA. PCR products sizes are indicated. (B) Southern blot screening of 6 ES cell clones for pRosa26-DUX4 knock-in. DNA was digested with EcoRV and probed with the probe indicated in A. WT ROSA26 alleles produce an 11,517bp EcoRV band, while correctly targeted clones produced a 4,084-bp band. (C) Configuration of 2 DUX4 transcripts detected in induced TIC-DUX4 mice via nested 3' RACE RT-PCR. Thirty-five of 36 clones contained correctly spliced products, while 1 clone had a retained intron 1. All transcripts utilized the BGH polyA signal. (D) Western blot of DUX4 expression in TIC-DUX4 mouse tissue. $\mathrm{H}$, high dose tamoxifen; L, low dose tamoxifen; -, untreated. Positive control (+ ctrl) is protein extracted from CMV. DUX4-transfected HEK293 cells. (E) Wfdc3 CDNA, a biomarker of DUX4 in mice, was significantly increased 1,395- and 1,438-fold in high (H) and low (L) Tam- induced TIC-DUX4 muscles compared with untreated (U, sunflower oil) or WT control littermates (-), respectively. Data are measured from qPCR assays $(P<0.01$; 1 -way ANOVA with Tukey's multiple comparison test). $n=4$ for high Tam dose TIC-DUX4; $n=3$ for all other groups.

into the system, the ROSA26 promoter drove DUX4 expression (Figure 1A). We attempted to cross the DUX4 transgenic mice with 3 different Cre recombinase-expressing lines. The first line, the HSA-Cre mouse model $\mathrm{Tg}$ (ACTA1-cre)79Jme/J, used a muscle-specific human skeletal actin (HSA) promoter to drive Cre expression. The resulting crosses did not produce any double-positive pups (Supplemental Figure 1B). The second line, $\mathrm{Tg}$ (ACTA1-rtTA,tetO-cre)102Monk/J, utilized a tet-On system to control HSA promoter-driven Cre, which allowed for doxycycline-inducible Cre expression. These crosses yielded only 2 double-positive pups that died on day P0 (Supplemental Figure 1B). The third line - Tg(ACTA1-cre/Esr1*)2Kesr/J, also called HSA-mER-Cre-mER or HSA-MCM, a Tam-inducible model — was ultimately used to establish 
the TIC-DUX4 double-transgenic line (28). HSA-mER-CRE-mER expressed a modified Cre recombinase protein fused with mutated estrogen receptors (mER) that served to allow sequestration of the protein in the cytoplasm via binding to the HSP90 protein complex. Tam administration interfered with this sequestration and allowed Cre to translocate into the nucleus and excise the floxed NeoR cassette that prevented DUX4 expression (Figure 1A). The expected result was DUX4 expression only in skeletal muscle, when and where the HSA promoter was active. The hemizygous HSA-mER-CRE-mER to heterozygous ROSA26-DUX4 crosses yielded double-transgenic pups in Mendelian ratios and were indistinguishable from single-transgenic or WT littermate controls. We called the double-transgenic line TIC-DUX4. The TIC-DUX4 mouse genotype was confirmed via PCR (Figure 1 and Supplemental Figure 1A). WT alleles and DUX4 knock-in alleles produced $573 \mathrm{bp}$ and $284 \mathrm{bp}$ PCR products, respectively. In addition, PCR for the mER-CRE-mER fusion resulted in a $717 \mathrm{bp}$ band when the Cre transgene was present (28) (Supplemental Figure 1A).

TIC-DUX4 express full-length DUX4. To confirm that full-length DUX4 transcript was produced upon Tam administration and determine if there was a preference for the pLAM or the BGH polyA signals, we isolated induced TIC-DUX4 mouse RNA from skeletal muscle, converted to cDNA, and performed nested 3' RACE. Sequencing of 3' RACE products revealed that the canonical BGH polyA signal was preferentially utilized (Figure 1C). Consistent with a previous study, this indicated that the pLAM PAS was inefficient and that read-through occurred into the BGH PAS to stabilize the transcript (22). Western blots confirmed that DUX4 full-length protein was present in induced skeletal muscles but not detected in uninduced muscle or in representative nonmuscle tissues (kidney or liver from induced mice) (Figure 1D). In addition, we used quantitative PCR (qPCR) to detect Wfdc3, a downstream target of DUX4 in mice, in a dose-responsive manner in induced TIC-DUX4 gastrocnemius (gas) muscle (Figure 1E). This demonstrated that DUX4 protein was functional as a transcription factor.

High-dose Tam-treated TIC-DUX4 mice show rapid onset of myopathic phenotypes. Two Tam administration routes were tested: Tam-laced chow and oral gavage. In addition, different doses were tested with each route (Table 1). TIC-DUX4 mice fed Tam-laced chow showed severe histopathology and significantly reduced activity within 2-3 weeks (Supplemental Figure 2, A-C). Muscle damage was evident in both upper- and lower-limb muscles. Degenerating fibers and immune cell infiltration colocalized with DUX4 expression (Supplemental Figure 2B). However, some TIC-DUX4 mice and WT controls did not adjust to the Tam food. The variability in food consumption created inconsistent Tam doses and phenotypes in both TIC-DUX4 and control mice, leading to death in some Tam-chow fed WT and TIC-DUX4 animals or significant loss of body mass (>20\%), requiring euthanasia in others before study end (Supplemental Figure 2, $\mathrm{D}$ and $\mathrm{E})$. These inconsistencies made Tam-laced chow a suboptimal method for DUX4 induction.

In contrast, the oral gavage method allowed more predictable control of Tam dose, which could be corrected according to body weight at each administration. We used 2 oral doses of Tam to turn on DUX4 expression in TIC-DUX4 mice: $150 \mathrm{mg} / \mathrm{kg}$ and $5 \mathrm{mg} / \mathrm{kg}$. The $150 \mathrm{mg} / \mathrm{kg}$ (high) Tam dose resulted in an acute-onset muscular phenotype within 7-9 days after induction. Severe muscle degeneration and immune cell infiltrates were observed throughout skeletal muscles (Supplemental Figure 2F). DUX4 expression was detected throughout the damaged muscle via immunofluorescence (Supplemental Figure 2G). The mice had a slow, unsteady gait, accompanied by a significant reduction in overall activity and whole muscle absolute and specific force compared with uninduced TIC-DUX4 controls (Supplemental Figure 2, H-K). High-dose Tam-treated TIC-DUX4 mice became nonambulatory 10 days after induction and required euthanasia per institutional endpoint criteria guidelines. The acute onset of the phenotype allowed for rapid DUX4 induction in muscle, but the large muscle lesions produced were inconsistent with progressive FSHD-like phenotypes and not useful for testing long-term treatments.

Tam dose deescalation produced less severe myopathic phenotypes in TIC-DUX4 mice. Importantly, reducing the Tam dose attenuated the muscle damage phenotype. Mice in the medium dose treatment group ( $5 \mathrm{mg} / \mathrm{kg} ; 3 \times /$ week) developed an observable gait change within 3 weeks after induction. At 4 weeks (endpoint for this course of Tam), induced TIC-DUX4 mice showed histopathological damage throughout the tibialis anterior (TA), gastrocnemius (gas), quadriceps (quad), and triceps (tri) as compared with uninduced TIC-DUX4 and WT controls (Figure 2 and Supplemental Figure 4). Both male and female TIC-DUX4 mice showed significant increases in myofibers containing centralized nuclei and a shift toward small fiber diameters, indicating that muscles were damaged and underwent regeneration (Figure 2, A and B, Supplemental Figures 3-5, and Supplemental Tables 1 and 2). This course of Tam treatment also resulted in functional deficits. Significant reductions in total activity and 
Table 1. Summary of tamoxifen doses administered to TIC-DUX4 mice

\begin{tabular}{|c|c|c|c|c|}
\hline Tamoxifen dose & Administration & Endpoints & Phenotype & Histopathology \\
\hline $150 \mathrm{mg} / \mathrm{kg}$ & $2 \times($ Day 0 and 2$)$ & 10 day & Severe & Severe \\
\hline $40 \mathrm{mg} / \mathrm{kg}$ & Ad libitum & 2 weeks & Severe & Moderate-severe \\
\hline $40 \mathrm{mg} / \mathrm{kg}$ & 2 days on diet/2 days off diet & 2 week & Severe & Moderate-severe \\
\hline 5 mg/kg & $3 \times /$ week & 4 week & Moderate & Mild-moderate \\
\hline $5 \mathrm{mg} / \mathrm{kg}$ & $1 \times$ week & 4 weeks -4 months & None-moderate & None-severe \\
\hline $1 \mathrm{mg} / \mathrm{kg}$ & $3 \times /$ week & 2 weeks or 5 weeks & None & None \\
\hline $5 \mathrm{mg} / \mathrm{kg} 3 \times /$ week & 2 week & None & None & - \\
\hline $5 \mathrm{mg} / \mathrm{kg} 2 \times /$ week & 2 weeks or 5 weeks & None & None & - \\
\hline
\end{tabular}

Induced phenotype summary based on 6- to 12-week-old mice induced at the dose and time point indicated. Doses of $40 \mathrm{mg} / \mathrm{kg}$ were Tamoxifen-laced chow. All other doses were administered by oral gavage.

rearing frequency indicated total body muscle weakness compared with uninduced TIC-DUX4 controls (Figure 3A). In addition, Tam-treated TIC-DUX4 TA muscles were significantly weaker (Figure 3B). Outcome measures in both activity and muscle strength appeared consistent between both sexes, although activity could vary greatly in the less-severe phenotypes (Figure 3). At this 12- to 15-week-old time point, age-matched, uninduced TIC-DUX4 mice did not demonstrate any significant histological or functional muscle deficits when compared with age-matched WT littermates and showed no detectable DUX4 protein expression without Tam induction.

The clinical description of FSHD does not typically include respiratory insufficiency or diaphragm involvement, although such deficits are reported in some patients. We therefore analyzed DUX4 expression and pathology in diaphragms of TIC-DUX4 mice and controls. Interestingly, although all Tam-induced mice examined had diaphragm expression and nuclear localization of DUX4, we observed variable diaphragm pathology. Some diaphragms displayed DUX4+ lesions; however, most fibers appeared normal, despite having DUX4+ ${ }^{+}$staining nuclei throughout the diaphragm (Figure $4 \mathrm{~A}$, first and second rows). As with limb muscles, DUX4 was not detected in uninduced animals via immunofluorescence staining or Western blot (Figure 1D and Figure 4A, third row). Of the 18 animals we examined, 61\% (11) had normal to near-normal diaphragm histology (defined as less than $5 \%$ central nuclei and normal fiber diameter distribution) (Figure 4, B and C; Supplemental Figure 5; and Supplemental Tables 1 and 2). Five animals ( 2 males, 3 females) displayed diaphragm myofibers with $>10 \%$ central nuclei, with 1 male having $28 \%$. Absolute and specific force in induced male and female TIC-DUX4 mice did not significantly differ from uninduced TIC-DUX4 mice or age-matched WT littermates (Figure 4D). These data suggested that the diaphragms may have some higher tolerance to DUX4 expression.

Because FSHD is often slowly progressive, we next investigated if we could attenuate the muscle damage phenotypes even further and extend the amount of time we could study induced mice past 4 weeks. We began by treating another cohort of animals with a lower Tam dose $(0.5 \mathrm{mg} / \mathrm{kg}, 3 \times$ per week for 5 weeks), but these animals showed no obvious histopathology (not shown) and had normal TA absolute and specific force when compared with controls (Figure 3B). In a second strategy, we continued to use the $5 \mathrm{mg} / \mathrm{kg}$ Tam dose but reduced the frequency of administration from $3 \times$ to once per week. Mice were treated with this Tam regimen for 1-4 months (Figure 5A and Supplemental Figures 6 and 7). At 1 month, Tam-induced TIC-DUX4 limb muscles showed mild, sporadic lesions, including some fibers with reduced eosin staining (suggesting degeneration) and rare central nuclei. These histopathological features were more evident at 2 months, with greater abundance of myofibers with central nuclei and the presence of immune infiltrations colocalized with DUX4 expression (Supplemental Figure 6). The severity in the phenotype increased dramatically at 3 and 4 months after induction, with many fibers having central nuclei and an observable increase in lesions present throughout the muscles (Figure 5A). Total activity and rearing behavior began decreasing by 1 month and were significantly reduced at the 2-month time point, followed by recovery to WT levels at the 3-and 4-month time points (Figure 5B and Supplemental Figure 7). This recovery may reflect an accumulation of muscle damage up to 2 months followed by regeneration and repair. We did not determine if a second wave of DUX4-induced damage would occur with chronic Tam induction beyond the fourth month of treatment. We measured gas and 

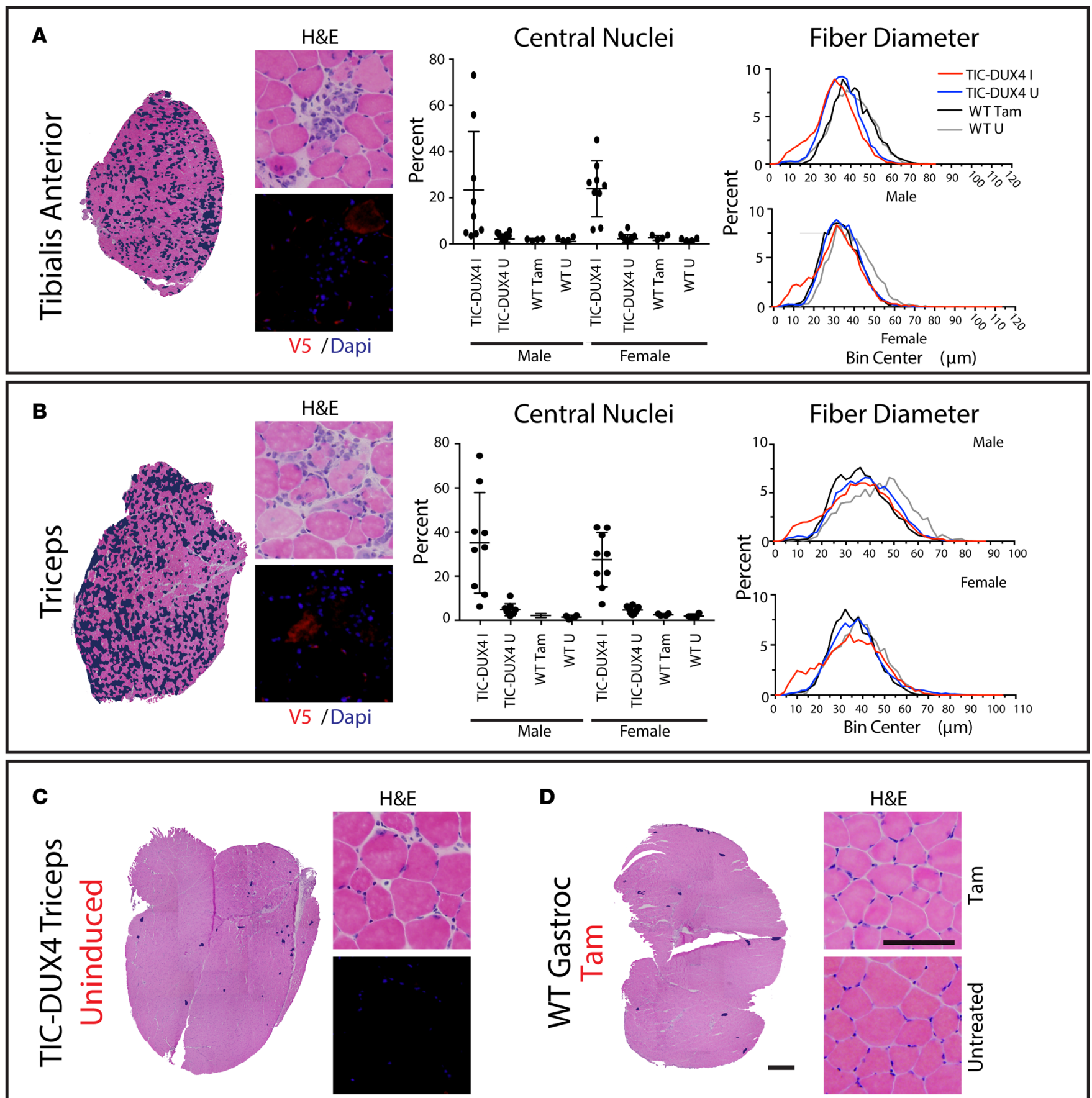

V5 /Dapi

Figure 2. Histopathology in TIC-DUX4 mice following a medium-dose tamoxifen regimen. (A and B) Left panels show tibialis anterior (TA) and triceps muscles from TIC-DUX4 mice 4 weeks after induction with $5 \mathrm{mg} / \mathrm{kg}$ tamoxifen, 3 times per week. Far left images show representative 10× muscle cryosections tiled to display an entire muscle in cross-section. Navy blue coloring was digitally added to each low-power image to easily visualize histopathology, including degenerating myofibers, regenerated myofibers containing central nuclei, and immune infiltration, which are features evident in the adjacent 20x images. The bottom higher-power images show immunofluorescence for DUX4 (V5 epitope, red) or nuclei (DAPI, blue). Widespread damage was present throughout induced TIC-DUX4 muscles. Increased percentage of myofibers with central nuclei and a shift toward smaller bore myofibers in induced TIC-DUX4 muscles support that muscles underwent degeneration and subsequent regeneration during this time course. Tamoxifen-induced TIC-DUX4 mice (TIC-DUX4 I) were significantly different from uninduced TIC-DUX4 (TIC-DUX4 U) and WT animals (2-way ANOVA with Tukey's multiple comparison test; $P<0.05$ for TA and $P<0.01$ for triceps). No significant sex differences were measured in induced TIC-DUX4 animals. Mean central nuclei percentages for TA muscle of induced TIC-DUX4 males: 23.4\%; uninduced TIC-DUX4 mice, 2.2\%; WT, Tam-treated 2.0\%; WT untreated, 1.1\%. For female TA muscles: induced TIC-DUX4 23.9\%; uninduced TIC-DUX4, 2.3\%; WT, Tam-treated 2.6\%; WT untreated, 1.3\%. For triceps, mean central nucleic percentages in induced TIC-DUX4 males: 34.8\%; uninduced TIC-DUX4, 4.5\%; WT, Tam-treated 1.9\%; WT untreated, 1.2\%. For induced TIC-DUX4 female triceps: 27.3\%; 
uninduced TIC-DUX4, 4.4\%; WT,Tam-treated 2.3\%; WT untreated 1.7\%. Animals were 8-12 weeks of age during this experiment. For male mice: $n=9$ induced and uninduced TIC-DUX4 mice; 4 tamoxifen-treated WT littermates; 4 untreated WT littermates. For females: $n=9$ induced and 10 uninduced TIC-DUX4 mice; 4 tamoxifen-treated WT littermates; 4 untreated WT littermates. (C) Representative uninduced triceps muscle of 8- to 12-week-old TICDUX4 mice. (D) Representative tiled 10x image showing tamoxifen-treated gas muscle of 8- to 12-week-old WT mice. 20x H\&E image (top right) demonstrated that tamoxifen treatment alone has no impact on muscle histology. Scale bars: $500 \mu \mathrm{m}$ in low-power images; $100 \mu \mathrm{m}$ at $20 \times$.

TA muscle strength at the 2 month time point and found that absolute force was significantly reduced compared with uninduced TIC-DUX4 mice. Specific force was significantly reduced in the gas compared with uninduced TIC-DUX4 controls; however, the reduction in the TA was not significant when compared with uninduced TIC-DUX4 controls (Figure 5, C and D).

Phenotypes in old, uninduced TIC-DUX4 mice. Uninduced animals showed no obvious functional deficits or detectable DUX4 expression in any prior experiment where animals were 12-25 weeks of age. However, we found that old uninduced mice ( $>1.5$ years) had increased levels of central nuclei and sporadic spots of immune cell infiltration, which indicated that myofibers had undergone some degeneration and subsequent regeneration (Supplemental Figure 8, A-E). In some cases, these histopathological lesions colocalized with sporadic DUX4 expression (Supplemental Figure 8, C and D). Functional tests revealed that 1.5-year-old mice had normal activity and rearing levels compared with age-matched WT littermate controls (Supplemental Figure 8, F and G). TA muscles of old, uninduced TIC-DUX4 mice were 33\% smaller than those of age-matched WT controls, and these smaller muscles showed significantly reduced absolute force output when compared with 1.5-year-old, age-matched WT controls, but were no different from 4-mo old WT controls (Supplemental Figure 8, H and I). There were no significant differences in body weight between 1.5-year-old TIC-DUX4 mice and WT controls (Supplemental Figure 8J). When absolute force was normalized to muscle cross-sectional area (i.e., specific force), force production per $\mathrm{mm}^{2}$ of muscle in uninduced, 1.5-year-old TIC-DUX4 mice was not significantly different from that of either WT control group (Supplemental Figure $8 \mathrm{H}$ ). We also measured activation of the DUX4-responsive murine gene, Wfdc3, in old TIC-DUX4 muscle and found a significant increase in relative expression compared with age-matched littermate controls. This supported that DUX4 expression did occur as the animals aged, although to a lesser exent than the Tam-treated TIC-DUX4 mice (Figure 1E).

AAV1.Follistatin increased muscle mass and absolute strength in induced TIC-DUX4 mice. We next sought to demonstrate that the myopathic phenotypes caused by DUX4 expression in TIC-DUX4 mice were useful as therapeutic outcome measures. To do this, we tested a myostatin inhibition gene therapy approach, which relied upon AAV1 delivery of the Follistatin isoform FS344. This promising strategy has been shown to improve strength in preclinical models of muscular dystrophy and has proven safe in human clinical trials for Becker muscular dystrophy and inclusion body myositis (29-34). As AAV1.Follistatin has been shown to improve muscle mass and strength in WT and disease animal models, we hypothesized that it could achieve similar effects in TIC-DUX4 mice but would likely not mitigate the damaging effects of DUX4 on individual myofibers $(30,31,34)$. To test this, we i.m. injected adult TIC-DUX4 mice with $1 \times 10^{11}$ DNase particles of AAV1.Follistatin in the TA and gas muscles. Mice were treated with oral Tam 1 week later ( $5 \mathrm{mg} / \mathrm{kg}$; $1 \times$ per week) for 8 weeks. We then measured TA and gas total force production and muscle weight. Because we performed localized i.m. injections and did not use a systemic delivery strategy, we did not measure rearing or total activity behavior. Compared with untreated TIC-DUX4 and C57BL/ 6 controls, induced TIC-DUX4 mice treated with Follistatin had significantly increased overall TA and gas size (Figure 6, A and C, and Supplemental Figure 9, A and C). Muscle histology remained unchanged in Follistatin-treated, induced TIC-DUX4 mice compared with untreated, induced TIC-DUX4 mice. Specifically, regenerated and degenerating fibers were evident throughout both muscles in AAV1.Follistatin-injected TIC-DUX4 animals and were similar to untreated, induced TIC-DUX4 mice (Figure 6B and Supplemental Figure 9B). This histopathology suggested that AAV1. Follistatin did not impact DUX4 protein expression or prevent DUX4-associated muscle damage in this study. We then measured muscle strength in this cohort. TA and gas absolute force were significantly increased in follistatin-injected, Tam-induced TIC-DUX4 mice compared with untreated, Tam-induced TIC-DUX4 mice (Figure 6D and Supplemental Figure 9D). In both muscles, specific force was not significantly improved due to the larger cross-sectional area of the AAV1.Follistatin-treated muscles (Figure $6 \mathrm{E}$ and Supplemental Figure 9E). However, the gas specific force may show an intermediate phenotype, as it was not significantly different from the WT controls. Overall, these results supported that AAV1. 
A Total Activity

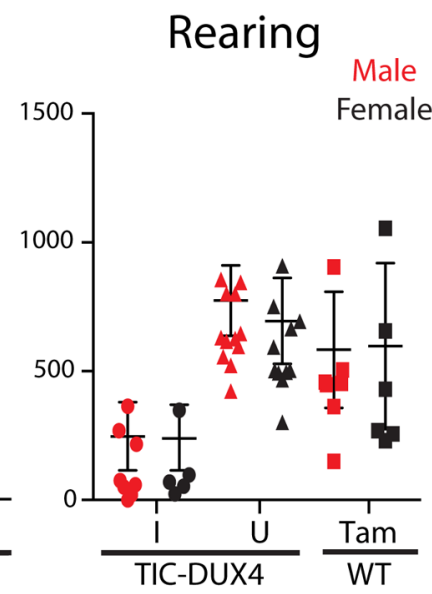

B TA Absolute Force

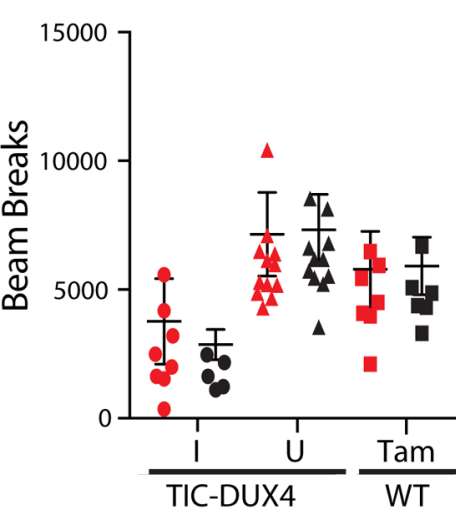

TA Specific Force
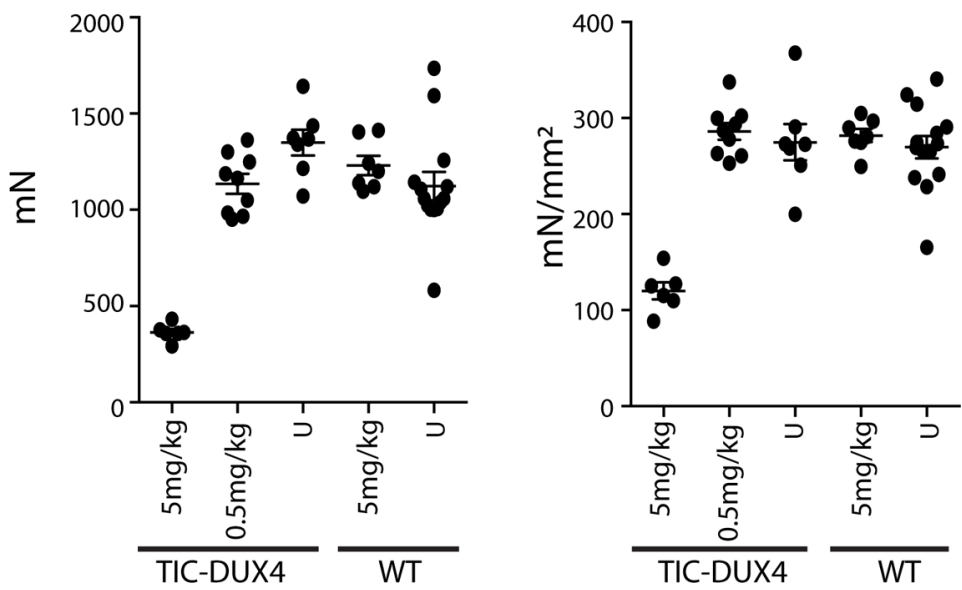

Figure 3. Functional deficits in TIC-DUX4 mice following a medium-dose tamoxifen regimen. (A) TIC-DUX4 animals treated with $5 \mathrm{mg} / \mathrm{kg}$ tamoxifen, $3 \times$ per week for 4 weeks showed significantly reduced overall cage activity and rearing behavior compared with vehicle (untreated) or WT controls. Induced TIC-DUX4 male and female mice had a mean activity of 2,616 and 1,719 mean beam breaks, respectively, compared with 5,999 and 6,173 mean beam breaks in uninduced TIC-DUX4 mice and 4,646 and 4,770 mean beam breaks in WT mice, respectively. Induced mice had a rearing frequency mean of 131.9 (male) and 124.3 (female) beam breaks compared with 659.9 (male) and 580.4 (female) beam breaks in uninduced TIC-DUX4 mice. WT animals had 468.6 (male) and 482.8 (female) mean beam breaks. Error bars show \pm SD. I, tamoxifen induced; U, untreated. $n=8$ induced male and 5 induced female TIC-DUX4 mice; 12 male and 11 female uninduced TIC-DUX4 mice; and 7 male and 6 female tamoxifen-treated WT littermate controls. For both total activity and rearing, induced TIC-DUX4 animals were significantly different from all control groups (2-way ANOVA with Tukey's multiple comparison test; $P<0.05$ ). (B) TIC-DUX4 mice treated with $5 \mathrm{mg} / \mathrm{kg}$ tamoxifen, $3 \times$ per week for 4 weeks developed significant weakness in the tibialis anterior muscles, as measured by absolute and specific force reductions compared with all control groups $(P<0.0001$, 2 -way ANOVA with Tukey's multiple comparison test). Animals treated with 10 -fold less tamoxifen concentration $(0.5 \mathrm{mg} / \mathrm{kg})$ during the same time course showed no measurable deficits. There were no significant differences between sexes, and data presented here are grouped only by treatment and genotype. Error bars represent \pm SD. Mean absolute force: TIC-DUX4 mice ( $5 \mathrm{mg} / \mathrm{kg} \mathrm{Tam}), 363.6 \mathrm{mN}$; TIC-DUX4 mice $(0.5 \mathrm{mg} / \mathrm{kg} \mathrm{Tam}), 1,135 \mathrm{mN}$; uninduced TIC-DUX4 mice, 1,349 mN; WT (5 mg/kg Tam), 1,231 mN; WT untreated, 1,125 mN. Mean specific force: TIC-DUX4 mice (5 mg/ $\mathrm{kg} \mathrm{Tam}), 120 \mathrm{mN} / \mathrm{mm}^{2}$; TIC-DUX4 (0.5 mg/kg Tam), $286.1 \mathrm{mN} / \mathrm{mm}^{2}$; uninduced TIC-DUX4 mice, $274.9 \mathrm{mN} / \mathrm{mm}^{2} ;$ WT ( $5 \mathrm{mg} / \mathrm{kg} \mathrm{Tam}), 281.8 \mathrm{mN} / \mathrm{mm}^{2} ;$ WT untreated, $269.7 \mathrm{mN} / \mathrm{mm}^{2} . n=6(5 \mathrm{mg} / \mathrm{kg})$ induced TIC-DUX4 legs; 9 (0.5mg/ kg) induced TIC-DUX4 legs; 7 uninduced TIC-DUX4 legs; 7 (5mg/kg) tamoxifen-treated WT littermate legs; and 14 untreated $\mathrm{WT}$ littermate legs. $\mathrm{mN}=$ milliNewtons.

Follistatin treatment could improve strength in DUX4-expressing muscles that would otherwise be weaker than controls and that this significant strengthening of treated muscles was likely due to muscle hypertrophy. These results suggested that myostatin inhibition may be an important treatment option for FSHD, even in the absence of DUX4-modulating therapies. 
$\mathbf{A}$

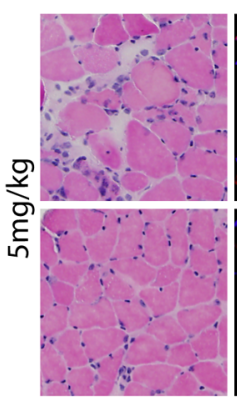

Male
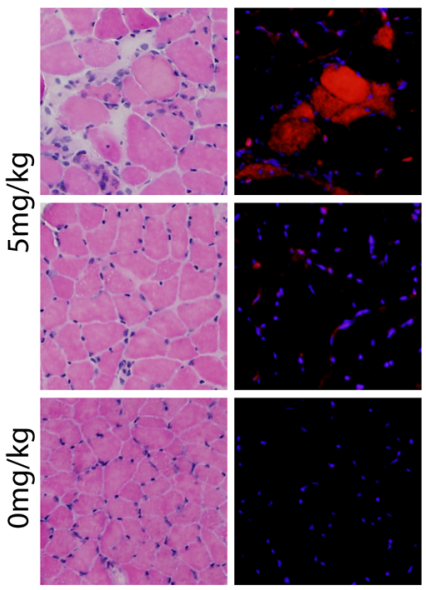

WT Littermates
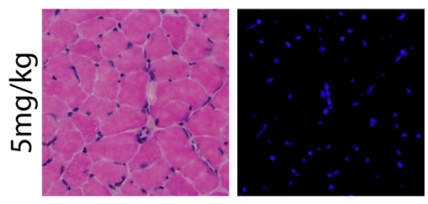

C

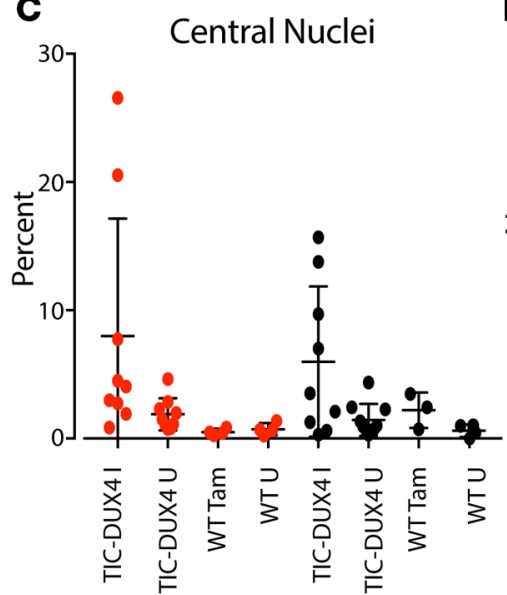

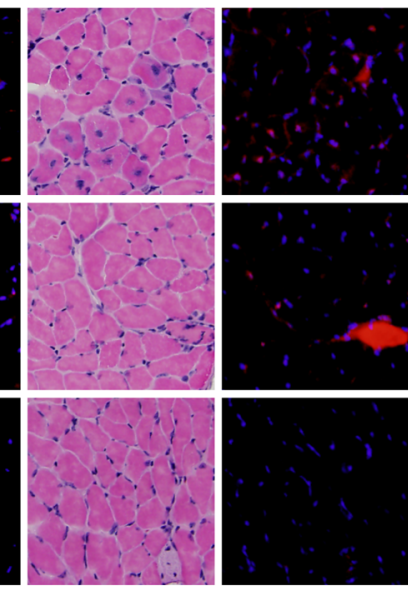

Female

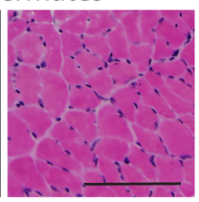

D

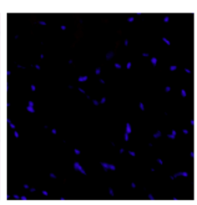

B

Absolute Force

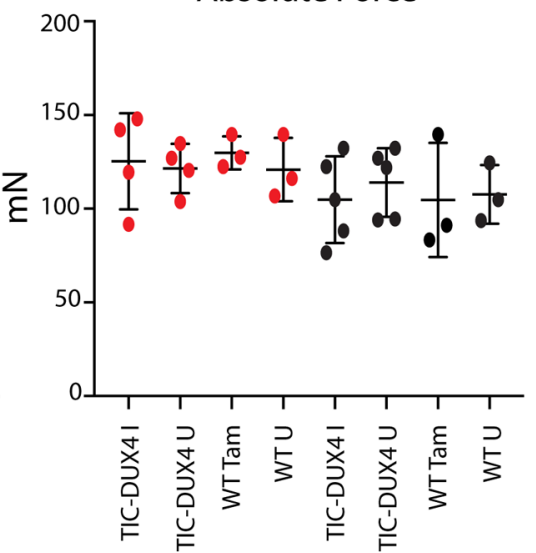

Fiber Diameter

Male

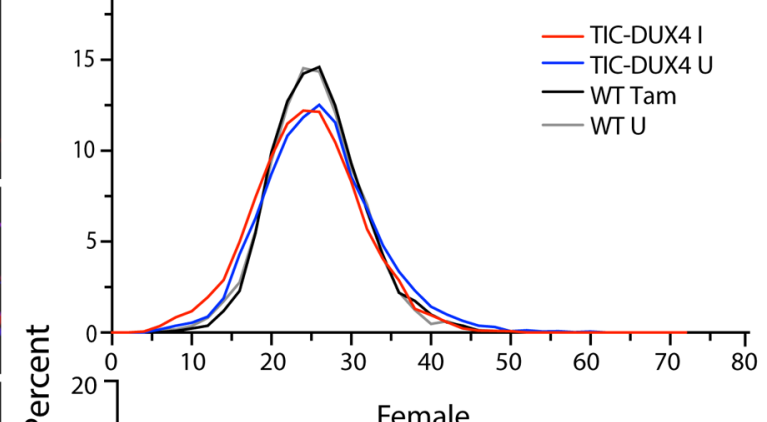

Female

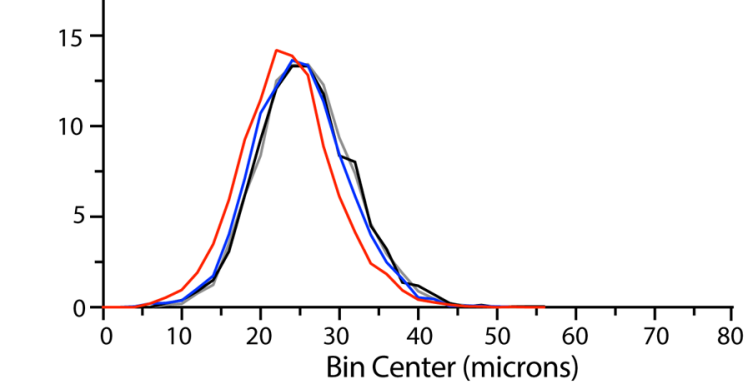

Specific Force

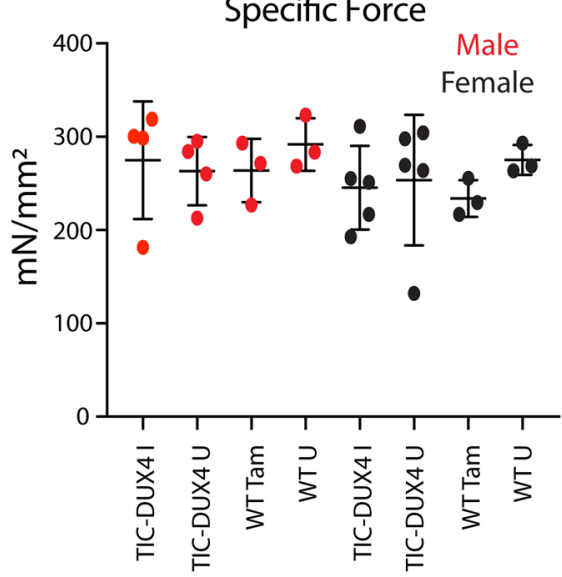

Figure 4. Variable histopathology and no significant functional deficits in TIC-DUX4 diaphragm muscles following a medium-dose tamoxifen regimen. Animals were treated with $5 \mathrm{mg} / \mathrm{kg}$ tamoxifen, or sunflower oil vehicle (U), $3 \times$ per week for 4 weeks. (A) Muscle cryosections from mice treated with indicated tamoxifen doses were stained with H\&E and immunofluorescence for DUX4 (V5 epitope, red) or nuclei (DAPI, blue). The top 2 rows show the range of histopathology seen in diaphragm muscles within this cohort. First row shows examples of tamoxifen-induced TICDUX4 diaphragms displaying histopathology (mild inflammation, central nuclei, reduced eosin staining) colocalizing with DUX4 protein labeling. DUX4 can be visualized in the nucleus of intact myofibers, as well as in the cytoplasm of cells showing decreased eosinophilic staining. Second row shows examples of tamoxifen-induced TIC-DUX4 diaphragms with few or no degenerating fibers, despite having DUX4-positive nuclei. Third row, uninduced TIC-DUX4 diaphragms do not stain positive for DUX4 protein and have normal histology. Fourth row, tamoxifen-treated WT littermate diaphragms do not stain positive for DUX4 protein and have normal histology. Magnification, 20x. Scale bar: $100 \mu \mathrm{m}$. (B) Male and female induced TIC-DUX4 diaphragm fiber size distributions overlap with those of uninduced TIC-DUX4 controls and WT littermates. (C) Scatter plot showing that male and female tamoxifen-induced TIC-DUX4 diaphragms have myofibers with variable central nuclei (CN) percentages. The mean CN percentages were increased in TIC-DUX4 mice induced with tamoxifen (males, $8 \%$ mean CN with $0.8 \%-26.6 \%$ range; females, $6 \%$ mean $\mathrm{CN}$ with $0.3 \%-15.7 \%$ range), but values were not significantly different from controls (all uninduced TIC-DUX4 or WT controls groups showed $<2.1 \%$ CN; 2 -way ANOVA with Tukey's repeated measures test). Nevertheless, 5 tamoxifen-induced TIC-DUX4 animals showed elevated CN above 10\%, topping out at $28 \%$ in 1 male. Males, $n=9$ induced and uninduced TIC-DUX4 mice; 4 tamoxifen-treated WT littermates; 4 uninduced WT littermates. Females $n=9$ induced and 10 uninduced TIC-DUX4 mice; 4 tamoxifen-treated WT littermates; 4 untreated WT littermates. (D) Diaphragm contractile force was normal in tamoxifen-induced TIC-DUX4 animals. Six-month-old mice were treated with $5 \mathrm{mg} / \mathrm{kg}$ tamoxifen $3 \times$ per week for 4 weeks. Plots show absolute force and specific force (absolute force normalized to muscle cross sectional area). $n=4$ male and 4 female tamoxifen-induced TIC-DUX4 animals; 4 male and 4 female uninduced TIC-DUX4 animals; 3 tamoxifen-treated WT littermates; and 3 uninduced WT littermate muscles. No significant changes; 2-way ANOVA with Tukey's multiple comparisons test. 
A

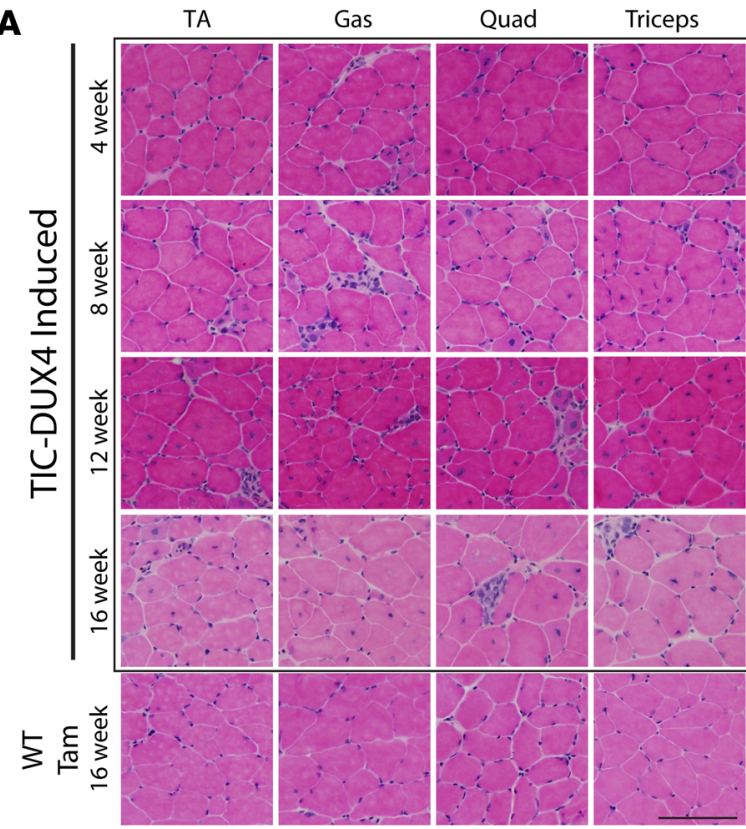

C

TA Absolute Force

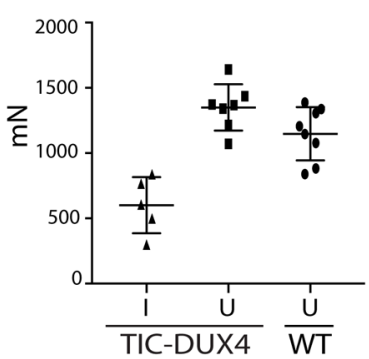

TA Specific Force

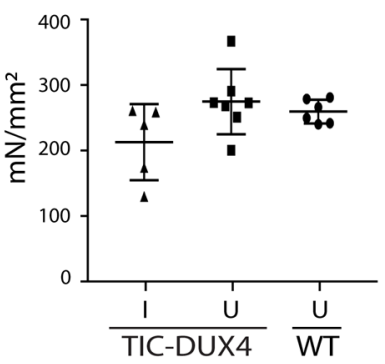

B
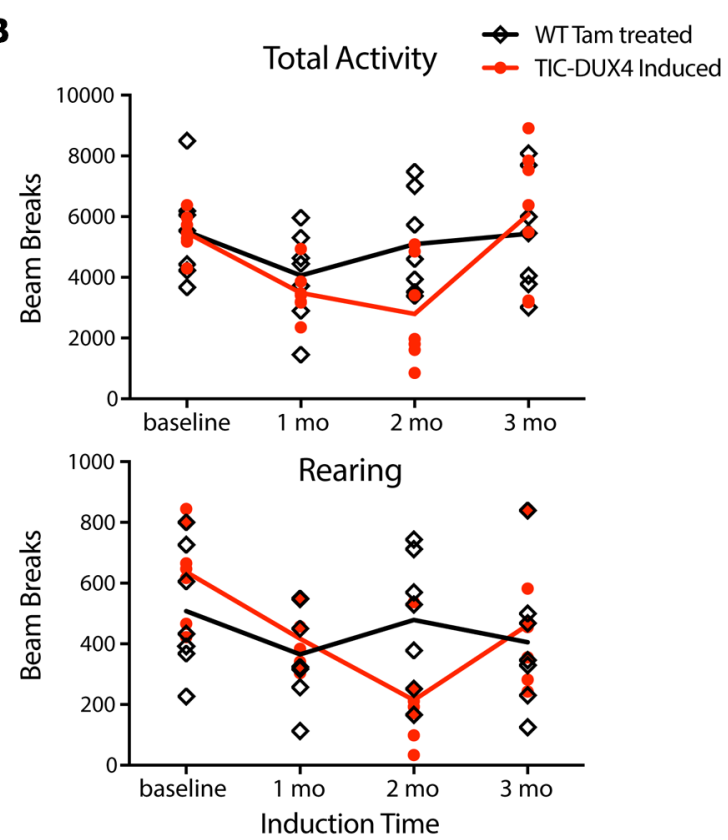

D

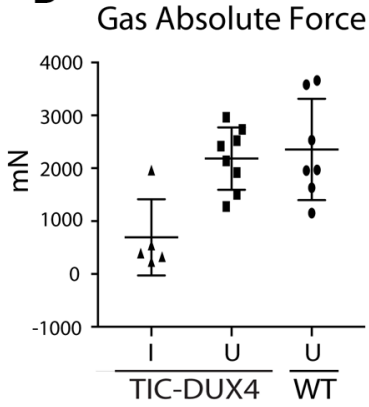

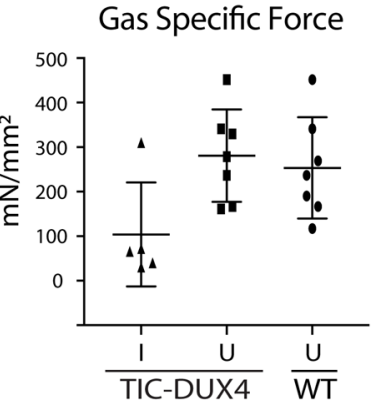

Figure 5. Progressive pathology in TIC-DUX4 mice treated with a low-dose tamoxifen regimen. Data for this figure were generated with 8- to 12-week-old mice induced with $5 \mathrm{mg} / \mathrm{kg}$ tamoxifen given 1x/week for 1-4 months, as indicated. (A) H\&E-stained cryosections (20x) from indicated muscles of TIC-DUX4 mice induced for 4 weeks to 16 weeks show progressive muscle degeneration and regeneration with time. WT littermates were treated with this tamoxifen regimen for 16 weeks and show normal muscle histology. (B) Total activity and rearing behavior were measured in all mice throughout the study. Top graph shows total cage activity from tamoxifen-treated TIC-DUX4 mice and WT littermates mice, measured monthly for 3 months, where baseline represents a pretreatment data point. The bottom graph shows rearing frequency in the same cohort over the same time course. There was a significant and progressive decline in Tam-induced TIC-DUX4 mouse activity and rearing that reached significance at 1 and 2 months, compared with TIC-DUX4 baseline (2-way ANOVA repeated measures; $P<0.05$ ). In contrast, activity and rearing behavior in WT animals was not significantly different across the time course. $n=7$ animal per group. When comparing TIC-DUX4 with WT animals at each time point, there was a significant difference between the groups only at the 2 month time point, for both overall activity and rearing behavior (Tukey's post-hoc test; $P<0.01$ ) (C and $\mathbf{D})$ Low-dose Tam treatment caused significant absolute force deficits in the TA $(P<0.0001)$ and gas $(P<0.01)$ muscles and significantly decreased specific force in the gas $(P<0.05)$ compared with uninduced TIC-DUX4 mice. Test results were obtained with 1-way ANOVA using Tukey's multiple comparisons test. For TA absolute force: Induced TIC-DUX4 mice, 601.3 mN; uninduced TIC-DUX4, 1,349 mN; WT, 1,148 mN). TA specific force: Induced TIC-DUX4 mice, $212.2 \mathrm{mN} / \mathrm{mm}^{2}$; uninduced TIC-DUX4, 274.9 mN/mm²; WT, 259.4 $\mathrm{mN} / \mathrm{mm}^{2}$. For gas absolute force: Induced TIC-DUX4 mice, $693.6 \mathrm{mN}$; uninduced TIC-DUX4, 2,184 mN; WT, 2,356 mN). Gas specific force: Induced TIC-DUX4 mice, $103.9 \mathrm{mN} / \mathrm{mm}^{2}$; uninduced TIC-DUX4, $280.9 \mathrm{mN} / \mathrm{mm}^{2}$; WT, $253.3 \mathrm{mN} / \mathrm{mm}^{2}$. For TA muscles, $n=5$ tamoxifen-induced TIC-DUX4 legs, 7 uninduced TICDUX4 legs, and 8 WT littermate legs. For gas, $n=5$ tamoxifen-induced TIC-DUX4 legs, 8 uninduced legs, and 7 WT littermate legs.

\section{Discussion}

Animal and cell model development has been a primary focus of the FSHD field and our laboratory since the DUX4 gene emerged more than a decade ago (35). During the last decade or so, several DUX4-expressing models have also been reported $(6,9,19-26,35-46)$. One particularly important study that guided the design of our TIC-DUX4 mice was first reported as a scientific abstract in 2008 and published in 2014 $(25,43)$. This work described a doxycycline-inducible DUX4 transgene knocked in to the Hprt locus on the mouse X-chromosome (iDUX[2.7] model). The authors reported animals with male-specific early lethality, absence of muscle phenotypes, and surviving X-inactivated female carriers with runted size and severe skin abnormalities. This important model demonstrated that even small amounts of DUX4 expression through 
A

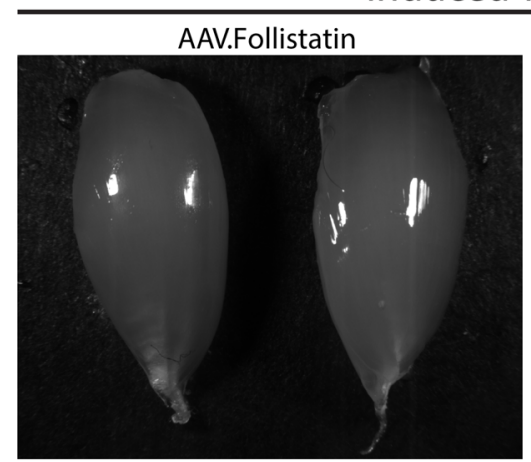

B

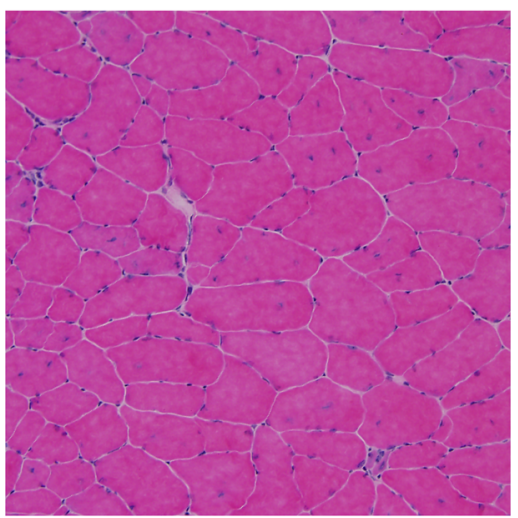

C

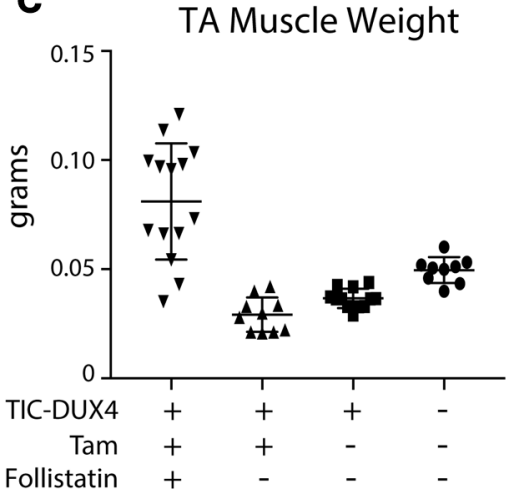

Induced TIC-DUX4
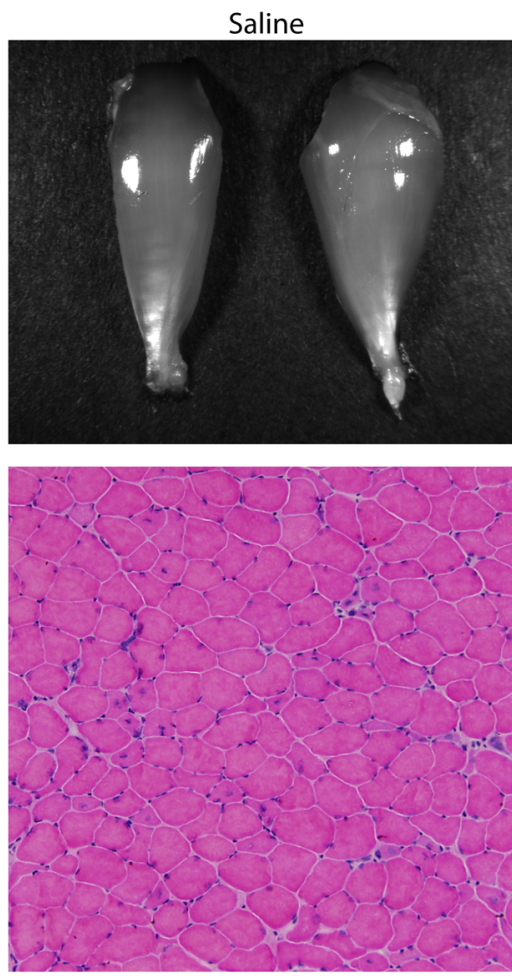

D

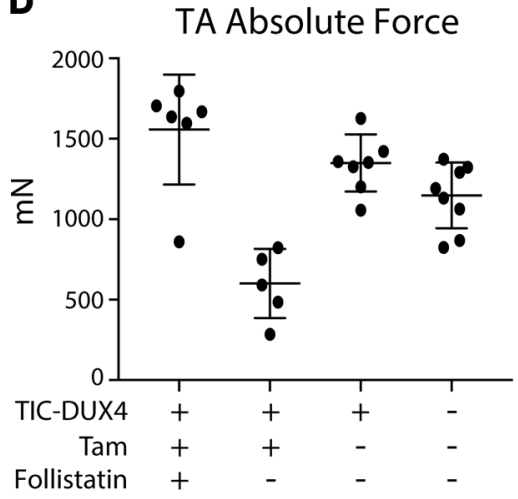

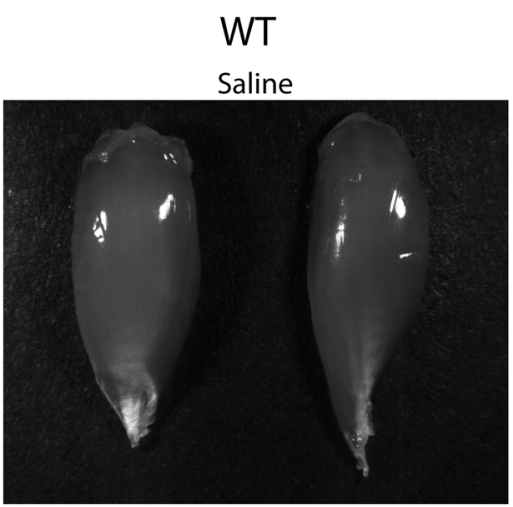

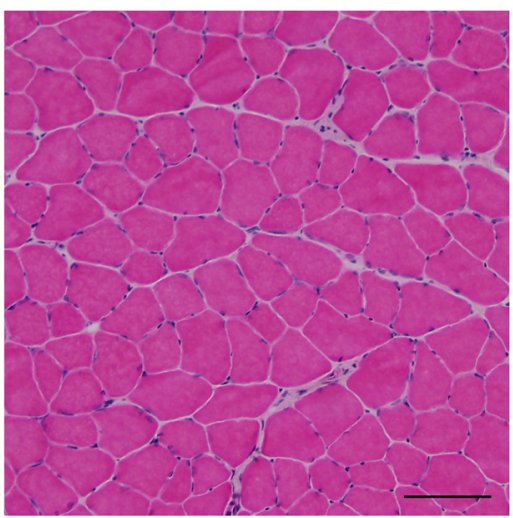

E

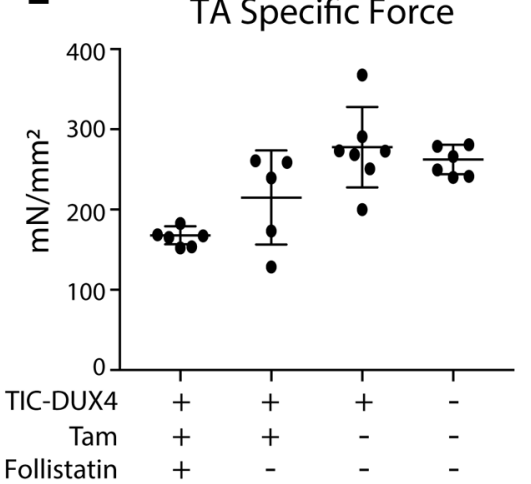

Figure 6. AAV1.Follistatin increases TA muscle mass and absolute force in the presence of DUX4 expression. (A) Six- to 7-week-old TIC-DUX4 and control mice were i.m. injected with $1 \times 10^{11}$ particles of AAV1.Follistatin. One week later, mice were treated with $5 \mathrm{mg} / \mathrm{kg}$ tamoxifen dosed $1 \times /$ week for 8 weeks. AAV1.Follistatin-injected TA muscles from tamoxifen-treated TIC-DUX4 were overtly larger than saline-injected TIC-DUX4 controls and WT muscles. Image magnification 4x. (B) H\&E-stained cryosections show evidence of histopathology (myofibers with central nuclei) in tamoxifen-treated TIC-DUX4 TA muscles expressing follistatin and saline injected TIC-DUX4 controls. Tamoxifen-treated WT controls injected with saline appeared normal. Scale bar: $100 \mu \mathrm{m}$. (C) Follistatin treatment significantly increased TA muscle weight compared with untreated controls (1-way ANOVA with Tukey's multiple comparisons test; $P<0.001)$. Mean TA muscle weights: Follistatin-treated, induced TIC-DUX4 mice, 81 mg; saline-treated induced TIC-DUX4 mice, 29 mg; uninduced TIC-DUX4 mice, 37 mg; age-matched WT, 50 mg. $n=14$ AAV1.Follistatin-treated, tamoxifen-induced TIC-DUX4 muscles; 10 saline-treated, tamoxifen-induced TIC-DUX4 muscles; 12 uninduced TIC-DUX4 muscles; 9 untreated C57/BL6 controls. (D) TA absolute force was significantly increased in induced TIC-DUX4 mice treated with AAV1. Follistatin compared with induced, saline-treated TIC-DUX4 controls (1-way ANOVA with Tukey's multiple comparisons test; $P<0.0001$ ). Mean TA absolute force: Follistatin-treated, induced TIC-DUX4 mice, 1,558 mN; saline-treated induced TIC-DUX4 mice, $601.3 \mathrm{mN}$; uninduced TIC-DUX4 mice, 1,349 mN; age-matched WT, 1,148 mN. (E) Specific force was determined by normalizing absolute force to muscle cross-sectional area. Follistatin treatment in induced TIC-DUX4 mice did not significantly improve specific force compared with all groups (1-way ANOVA with Tukey's multiple comparisons test). $n=6$ AAV1.Follistatin-treated, tamoxifen-induced TICDUX4 muscles; 5 saline-treated, tamoxifen-induced TIC-DUX4 muscles; 7 uninduced TIC-DUX4 muscles; 8 untreated WT controls.

development could be harmful to mice and that tightly controlled DUX4 expression would be necessary to produce viable animals. Based on those crucial observations, around 2009, we began working to generate the TIC-DUX4 model. We cloned a targeting construct, performed in-house homologous recombination in ES cells, generated animals, and - in the second year of the project — discovered an inadvertent splicing 
defect that rendered the DUX4 gene nontoxic (47). This necessitated eliminating the first lines, repairing the targeting construct, rederiving new ES cells, and generating another set of animals, at which time we had to seek additional funding to support characterization. As noted, our initial attempts to generate mice with muscle-specific Cre-drivers were unsuccessful, supporting others' prior observations that tightly controlled DUX4 expression was required to produce the desired animals (Supplemental Figure 1) $(22,43,44)$. After many years, overall we believe we accomplished the goals of our study, as the ROSA26-DUX4 and TIC-DUX4 mice were reproductively viable, produced progeny in Mendelian ratios, showed no extramuscular deficits, and displayed significant Tam- and DUX4-dose dependent myopathic phenotypes.

At the histological and molecular levels, we observed Tam dose-dependent muscle damage in induced TIC-DUX4 mice that was associated with DUX4 expression. DUX4 was detectable at the genomic, transcript, and protein level. At the protein level, we had difficulty detecting DUX4 in Western blots, despite being tagged with a strongly immunogenic V5 epitope for which high-affinity antibodies were commercially available. Surprisingly, we required loading large amounts of total protein to detect relatively low DUX4 protein signals by Western blot, even with acute, high-dose $(150 \mathrm{mg} / \mathrm{kg})$ induction. At this point, we are uncertain why DUX4 protein expression was difficult to detect biochemically, but this phenomenon could be related to protein solubility problems and extraction methods may require additional optimization. In contrast, DUX4 protein was more readily visualized in induced muscle via V5 immunofluorescence. At lower Tam doses (5 $\mathrm{mg} / \mathrm{kg}$ ), we confirmed that DUX4 expression occurred in sporadic fibers that were actively degenerating. This sporadic nature was likely due to inefficient Cre-mediated excision of the inhibitory floxed-Neo cassette at lower Tam doses. Interestingly, this pathology correlated with the burst model of DUX4 expression in human patients, which suggested that bursts of high DUX4 expression occurred in rare fibers and caused those fibers to die rapidly and then undergo repair. It has been proposed that multiple damaging events eventually could lead to profound weakness in certain muscles over the course of many years in a human being with FSHD (10). Progressive damage also occurred in our Tam-induced TIC-DUX4 mice, although not on the years-long time scale seen in humans, as mice only live 2 years. Instead, the speed at which damage took place correlated with the Tam dosing regimen. Interestingly, our activity data suggested that there may be some periodicity to this pathology in mice, as animals treated with the $5 \mathrm{mg} / \mathrm{kg}$ dose of Tam, $1 \times$ per week showed progressive activity deficits up to 2 months and then recovered by months 3 and 4 . These data suggested that an accumulation of damaging events occurred that weakened the animals, but then subsequent repair events allowed for some recovery in these activity outcomes. This type of recovery is not typical of the disease course in FSHD patients, and we speculate that this could reflect a higher capacity for muscle regeneration and repair in the mouse. Additional studies need to be performed to determine if additional rounds of activity deficits are seen beyond 4 months and to explore possible mechanisms for this phenomenon.

It is likely that the Cre system utilized was the key to viability in our animals. This was underscored by the embryonic lethality we reported in the constitutive and doxycycline-Cre mice that we attempted to cross to the ROSA26-DUX4 mice (Supplemental Figure 1). We did detect some evidence of leaky DUX4 expression in old, uninduced TIC-DUX4 mice. Our uninduced TIC-DUX4 mice never developed overt muscle weakness or activity phenotypes, but we did note an accumulation of histological DUX4-associated damage, smaller muscles, activation of the DUX4-associated biomarker Wfdc3, and decreased overall force output in some uninduced TIC-DUX4 muscles older than 1.5 years. This supported that the Neo cassette placed upstream of the DUX4 ORF largely prevented the expression of DUX4 protein and avoided most of the pathologies produced upon its induction with Tam but that some milder phenotypes could emerge with age. Taken together, these data demonstrate TIC-DUX4 mice could be induced to produce a spectrum of damage phenotypes that correlated with DUX4 expression and that some mild phenotypes could be produced in very old mice without Tam-induction.

Concern has been raised about the relevancy of expressing DUX4 in a mouse system because of possible species-specific divergences in downstream targets of the DUX4 transcription factor. Nonetheless, DUX4-mediated pathology is still the key driver in FSHD in humans and in our TIC-DUX4 line, as well as in other published models $(22,25,35,44)$. Even if $D U X 4$ downstream networks are incompletely conserved in the mouse, DUX4 still caused muscle cell death, immune infiltration, and muscle weakness in DUX4 mouse models. This supports that DUX4-expressing mouse models are important tools in preclinical therapeutic development studies. Toward this end, we used this mouse model to test whether myostatin inhibition via AAV-delivered Follistatin could improve muscle strength deficits in induced TIC-DUX4 muscles. This strategy would not directly target DUX4, as we have previously described using RNA interference 
(RNAi) $(48,49)$, but we hypothesized that increasing muscle force output through myostatin inhibition could help counteract the weakness caused by DUX4 damage.

Myostatin is an endogenous inhibitor of muscle growth that is highly conserved between rodents and humans. This conservation suggested that myostatin inhibition strategies tested in rodents could translate to humans. Indeed, myostatin inhibition using gene therapy and non-gene therapy approaches has been successfully tested in several preclinical animal models of muscle disease, and these data were used to support several human clinical trials. Two published clinical studies supported by Wyeth (MYO-029) and Acceleron (ACE031) utilized recombinant antibodies or ligand trap proteins, respectively, to prevent myostatin from binding its endogenous receptors when delivered to patients $(50,51)$. Both trials were initiated as placebo-controlled, double-blind, dose-escalation studies with safety as the primary endpoint. In both cases, trials were designed to deliver the products through multiple injections every 2-4 weeks for several months. In the Wyeth study, MYO029 was reported to be well-tolerated and generally safe, but the authors reported that 12 patients in the highdose cohorts developed skin rash or hives, necessitating suspension of the highest-dose cohort. No significant improvements in muscle strength or function were found, although the study was not powered for efficacy. Similarly, no significant functional improvements and no serious adverse events were reported in the Acceleron trial, although the study was halted due to safety concerns related to the vasculature (nosebleeds and telangiectasias). Although both products were halted for further development, Acceleron is currently developing a next-generation molecule (ACE-083) that is currently being delivered to FSHD patients in a Phase II clinical trial.

In this study, we chose to test a preclinical gene therapy approach to inhibit myostatin with AAV-delivered follistatin in our TIC-DUX4 mice. AAV1.Follistatin was an attractive option for several reasons. First, AAV1. Follistatin has been proven safe in Phase I/IIa human clinical trials for patients with Becker muscular dystrophy and inclusion body myositis $(29,32)$. Second, Follistatin may contribute to improving muscle strength through several mechanisms and may therefore have a greater role in muscle regulation than solely inhibiting myostatin (52-54). Indeed, when Follistatin was genetically overexpressed, muscle size increased more when compared with myostatin-null mouse models (33). Third, a single dose of AAV-delivered Follistatin could express in muscle cells for years, thereby offering a potential one-time muscle-strengthening treatment, as opposed to nongene therapy methods that must be injected repeatedly and indefinitely to achieve functional improvements. Moreover, since Follistatin is a secreted protein, Follistatin gene therapy could offer benefit to even untransduced muscles. Finally, potential off-target effects of Follistatin can be mitigated by expressing an isoform of Follistatin (FS344; used here) that does not bind to cells in the reproductive system.

In our study, TIC-DUX4 animals treated with a single i.m. dose of AAV1.Follistatin to lower leg muscles had increased muscle size and, importantly, showed significant increases in total muscle force. This benefit was seen in the presence of DUX4 induction and is therefore a relevant test of AAV1.Follistatin treatment in FSHD disease where DUX4-modulating therapies are not yet available. In the next steps of this study, it will be important to test the ability of systemically delivered AAV1.Follistatin to mitigate activity and rearing outcomes in whole mice, in addition to the isolated muscle strength outcomes we reported here. It will also be interesting to test the impact of combining AAV1.Follistatin treatment with DUX4-inhibitory strategies, which could work to both suppress DUX4-associated damage and improve muscle mass and strength. In conclusion, this proof-of-principle study provided encouraging evidence that DUX4 expressing muscle can be treated with AAV-delivered myostatin inhibition approaches to improve muscle function. We conclude that TIC-DUX4 mice are a relevant model to study DUX4 pathogenicity and disease progression. Moreover, the TIC-DUX4 mouse develops numerous molecular, histological, and functional outcomes that can be used as powerful tools to test gene therapies and other therapeutic strategies for FSHD.

\section{Methods}

Rosa26-DUX4 targeting construct generation. The DUX4 ORF was PCR amplified with primers containing EcoRV sites (Forward primer, 5'-CACCGCTAGCCCACCATGGCCCTCCCGACAC-3', and reverse primer, 5'-AAGATATCATAAAGTGTAAAGCCTGGGCCACTATAGGGCGAATTGGG-3') gel purified, inserted into the blunt cloning vector $\mathrm{pENTR}-\mathrm{TOPO}$ (Invitrogen) and transformed into TOP10 competent cells (Invitrogen). The 3' UTR pLAM region was PCR amplified with forward primer 5'-CGTACGCGTACGGAAGTTCCTATTCTCTAGAAAGTATAGGAACTTCGACGCGGGGTTGGGACGGGG-3' and reverse primer 5'-CGTACGCGTACGCATAAAGTGTAAAGCCTGGGGAAGTTCCTATACTTTCTAGAGAATAGGAACTTCTATAGGATCCACAGGGAGGG-3'. 
Both primers added FRT sites and BsiWI restriction enzyme sites at the $5^{\prime}$ and $3^{\prime}$ end of the PCR product. This product was cut with BsiWI and ligated with pENTR-DUX4.V5 digested with Acc65I; it was then transformed into TOP10 competent cells. pENTR-DUX4.V5.FRT.pLAM was verified by BamHI digestion. The original V5 peptide tag was mutated to prevent mis-splicing of the V5 and DUX4 stop codon via recombinant PCR, as previously described and ligated into pENTR-DUX4.V5.FRT.pLAM (47).

pROSA26-DEST plasmid was grown in DB33.1 cells at $30^{\circ} \mathrm{C}$ and isolated via Qiagen Miniprep kit. pENTR-DUX4.V5.pLAM and pROSA26-DEST plasmids were recombined in the Gateway LR system according to manufacturer's protocol specifications and transformed into One-Shot Stbl3 (Invitrogen) cells, grown at $30^{\circ} \mathrm{C}$. This resulted in a final pROSA26-DUX4 plasmid construct that was confirmed by BamHI digestion and $\mathrm{KpnI} / \mathrm{NcoI}$ double-restriction digestion and DNA sequencing

Rosa26-DUX4 knock-in mouse generation. The targeting construct was electroporated into TC1 ES cells (129S6/SvEvTac) with a Gene Pulser II apparatus (Bio-Rad). A total of 288 G418-resistant clones were selected. DNA was extracted from each clone, digested with EcoRV, and used to perform Southern blot screening. The Southern probe sequence was: (5'-TCTTCTCAGCTACCTTTACACACCATTGCACCGCTCTTGCCCAGAGAGAAAGGCTCTCCTTCATCTAGTCGACCCCACTACСTTTTTAATGTCTTCCCTGGGTCAGGACTCTTCCССTCCCCCTACTCTGGTCTCCCСTTTTTGCCTGGGTATTGССTACTCCACGTTTATACCCTTTTCAGGAGAGGCCTCCCAACCCTGCTCTCAAAATACACATACTTTTTTTTCTGTCCCTGAGCCCCCCACCTCCCCTGTTCTTGCGGCCTTGTGACAACTCTGGTCGCTCGTGGGGGCCCAGTCCTCCCСTCCATAATCTTCCTGAACGCCTCTCCTCTGGTTTTCCAGTTCCTATCTCAGATGGCTGCTGCTTTTCCCACACCAAAGACATTACCTTCGCCACCCCCACCTCACATTCTTGGACTCCCTGTGGCGTATGCCCCAGTATCCTT-3'). Sixteen correctly targeted clones were identified, and 1 clone (A1) was used to generate knock-in mice. Chimera production included 3-week-old albino C57BL/6J-Tyr-2J females, which were superovulated by administering 5 international units (IU) pregnant mare serum gonadotropin (PMSG) i.p., followed by 5 IU human chorionic gonadotropin (HCG), 48 hours after PMSG. The mice were then mated with albino C57BL/6 males, and blastocysts were harvested from pregnant females at 3.5 days post-coitus (d.p.c.). To generate chimeras, 5-10 ES cells from 1 correctly targeted clone were injected into the albino C57BL/6 blastocysts, which were subsequently transferred into the uterus of 2.5 d.p.c. pseudopregnant ICR strain females. Chimeric males recognized by the presence of black pigmented coat color were then bred with C57BL/ 6 females. Their pups were then screened for the presence of the insertion by PCR, and positive males and females were then bred with C57BL/6 mice to establish a hemizygous colony.

Generation of TIC-DUX4 mouse and genotyping of mouse lines. Heterozygous Rosa26-DUX4 mice were crossed with heterozygous HSA-Cre mice Tg(ACTA1-Cre)79Jme/J (stock no. 006149)), doxycycline inducible HSA-Cre mice (ACTA1-rtTA,tetO-Cre)102Monk/J (stock no. 012433), or HSA-mER-CRE-mER mice $\mathrm{Tg}(\mathrm{ACTA} 1-C r e / E s r 1 *) 2 \mathrm{Kesr} / \mathrm{J}$ (stock no. 025750) obtained from the Jackson Laboratory. Only HSA-mERCRE-mER mice crossed with DUX4 mice produced healthy pups, and this line combined with Rosa26DUX4 animals yielded the TIC-DUX4 strain. The ROSA26-DUX4 mice are available at The Jackson Laboratory (stock no. 032779). The HSA-mER-CRE-mER Tg(ACTA1-Cre/Esr1*)2Kesr/J line utilizes the HSA promoter to drive expression of Cre recombinase attached to modified estrogen receptors. To determine mouse genotypes, genomic DNA was extracted in $75 \mu \mathrm{l}$ of $50 \mathrm{mM} \mathrm{NaOH}$ solution for 1 hour and neutralized with $33 \mu 1$ of $1 \mathrm{M}$ Tris, $\mathrm{pH}$ 7.6. The HSA-mER-CRE-mER mouse line was genotyped via PCR as previously described with forward primer 5'-GCATGGTGGAGATCTTTGA-3' and reverse primer 5'-GCTTCTGTCCG TTTGCCGGTCG-3' (28).

The DUX4 gene was detected using 3 primers so that homozygous and heterozygous genotypes could be distinguished. A common forward primer, 5'-AGCTGCAGTGGAGTAGGCG-3', was used with a WT binding reverse primer, 5'-GGAGCGGGAGAAATGGATATG-3', and DUX4 knock-in reverse primer, 5'-GCGAAGAGTTTGTCCTCAAC-3'. PCR conditions were $94^{\circ} \mathrm{C}$ for 5 minutes, followed by 34 cycles of $\left(94^{\circ} \mathrm{C}\right.$ for 15 seconds, $60^{\circ} \mathrm{C}$ for 30 seconds, and $72^{\circ} \mathrm{C}$ for 30 seconds), with a final extension of $72^{\circ} \mathrm{C}$ for 10 minutes. PCR from heterozygous mouse DNA resulted in one 600-bp amplified band from the WT allele and one 300-bp band from the DUX4 knock-in allele.

Tam dosing. To induce Cre recombinase, mice were treated with Tam (MilliporeSigma) (via 2 delivery routes. One route included Tam chow. Adult mice (6-8 weeks) were fed Tam-laced chow ( $250 \mathrm{mg} / \mathrm{kg}$ diet) (Envigo Teklad Diets) ad libitum until sacrificed at 2 weeks after induction. The final approximate dose was $40 \mathrm{mg} / \mathrm{kg} /$ day, assuming a 3-4 g food intake for a $25 \mathrm{~g}$ mouse daily. The second route included oral 
gavage. Tam was dissolved in $95 \%$ ethanol at $10 \%$ final volume and then diluted in sunflower seed oil to $15 \mathrm{mg} / \mathrm{ml}$ concentration. The $150 \mathrm{mg} / \mathrm{kg}$ dose was given to adult mice (6 weeks to 1 year), which received two $150 \mathrm{mg} / \mathrm{kg}$ Tam doses on day 0 and day 2 and were sacrificed 7-10 days after induction. The $5 \mathrm{mg} /$ $\mathrm{kg}$ dose involved Tam being further diluted in sunflower oil to a $5 \mathrm{mg} / \mathrm{ml}$ concentration. Mice were given $5 \mathrm{mg} / \mathrm{kg}$ Tam doses 1-3 days per week until sacrificed at 4-16 weeks after induction.

$3^{\prime} R A C E$ PCR sequencing of DUX4 transcript. RNA was extracted with Trizol according to manufacturers' conditions, except that the chloroform extraction and ethanol washes were repeated for a total of 2 times. cDNA was generated using SuperScript IV First-Strand Synthesis System (Invitrogen) using an oligo $\mathrm{d}(\mathrm{T})$ reverse primer containing an adaptor for the endogenous DUX4 poly-A signal 5'-GAATCGAGCACCAGTTACGCATGCCGAGGTCGACTTCCTAGATTTTTTTTTTTTTTTTTTT-3'.

For reverse transcription, $2 \mu \mathrm{g}$ of RNA was treated with dsDNase (Thermo Fisher Scientific) according to manufacturer's specifications. RNA was then incubated with reverse primers listed above at 2.7- $\mu \mathrm{m}$ final concentration at $80^{\circ} \mathrm{C}$ for 2 minutes, followed by $95^{\circ} \mathrm{C}$ for 2 minutes. Then $5 \times$ reverse transcription (RT) buffer, Superscript IV RT, and RNase inhibitor were added to reactions and incubated at $55^{\circ} \mathrm{C}$ for 15 minutes, $60^{\circ} \mathrm{C}$ for 15 minutes, and $65^{\circ} \mathrm{C}$ for 60 minutes. Reactions were then treated with $1 \mu 1$ of $\mathrm{RNase} \mathrm{H}$ at $37^{\circ} \mathrm{C}$ for 15 minutes. Then, cDNA was used in nested PCR performed with a first round of the following PCR primers: forward primer: 5' primer Dux4-F_Ex1(Nested1) CTCAGCGAGGAAGAATACC and 5' reverse primer: Adapter-Nested1 GAATCGAGCACCAGTTACGCATG a second round of PCR was performed with forwards primer: forward primer, 5'-CTCAGCGAGGAAGAATACC-3', and reverse primer, 5'-GAATCGAGCACCAGTTACGCATG-3'. A second round of PCR was performed with forward primer 5'-GAG GAA GAA TAC CGG GCT CTG-3' and reverse primer, 5'-GAGCACCAGTTACGCATGCC-3'. Final PCR products were inserted into TOPO Blunt and confirmed with sequencing.

qPCR. RNA and cDNA was processed as described above. qPCR was performed using mouse Wfdc3 Taqman primer/probes (Mm01243777_m1) according to manufacturer's protocol (Thermo Fisher Scientific). Taqman Rpl13 primer/probe (Mm02526700_g1) was used to amplify a reference gene for qPCR.

Western blotting. Muscle and organ tissue was harvested from animals at endpoints and was ground and lysed in T-PER Tissue Protein Extraction Reagent containing Pierce Protease Inhibitor Mini Tablets (Thermo Fisher Scientific). Total protein was quantified using the DC Protein Assay (Bio-Rad), and 200 $\mu \mathrm{g}$ was separated by electrophoresis through a $12 \%$ SDS-polyacrylamide gel with $5 \%$ stacking gel. Protein was then transferred to nitrocellulose. Membranes were blocked in 5\% milk for 1 hour at room temperature. Protein was visualized using anti-V5-HRP antibody (Invitrogen, R961-25) (1:5,000 dilution) incubated at $4^{\circ} \mathrm{C}$ overnight. Membranes were washed $3 \times$ for 15 minutes in $0.1 \%$ tween in TBST and then incubated with SuperSignal West Femto Maximum Sensitivity Substrate (Thermo Fisher Scientific). Loading controls were separated by electrophoresis through a 4-12\% SDS-polyacrylamide gel (Mini Protean TGX gel, Bio-Rad) and stained with BlueFast staining dye (Eton Bioscience) according to manufacturer's protocol.

Histological analysis. Skeletal muscles were dissected from mice at indicated times after induction for histological analysis. Muscles were then frozen in Tissue Tek OCT Compound in liquid-nitrogen cooled isopentane (Takura). Fresh frozen cryosections $(10 \mu \mathrm{m})$ were generated and H\&E stained as previously described (48). Fiber diameter and central nuclei quantifications were determined from muscles $(n=5$ muscles per group in C57BL/ 6 mice and 9 muscles per group in TIC-DUX4 mice; 4 representative $\times 20$ photomicrographs per section), using cellSens Standard Software (Olympus). Fiber size diameter histograms represented percentage of total fibers analyzed. Male TA, $n=11,218$ induced TIC-DX4 fibers; 9,457 uninduced TIC-DUX4 fibers; 3,309 Tam-treated WT fibers; and 3,415 untreated WT fibers. Female TA, $n=10,942$ induced TIC-DUX4 fibers; 11,300 uninduced TIC-DUX4 fibers; 4,132 Tam-treated WT fibers; 3,435 untreated WT fibers. Male gas, $n=11,114$ induced TIC-DX4 fibers; 8,962 uninduced TIC-DUX4 fibers; 3,886 Tam-treated WT fibers; and 3,785 untreated WT fibers. Female gas, $n=11,203$ induced TIC-DX4 fibers; 12,395 uninduced TICDUX4 fibers; 4,890 Tam-treated WT fibers; 4,506 untreated WT fibers. Male quad, $n=9,605$ induced TICDUX4 fibers; 7,229 uninduced TIC-DUX4 fibers; 2,825 Tam-treated WT fibers; 2,542 untreated WT fibers. Female quad, $n=8,955$ induced TIC-DUX4 fibers; 10,215 uninduced TIC-DUX4 fibers; 4,276 Tam-treated WT fibers; 3,298 untreated WT fibers. Male tri, $n=9,119$ induced TIC-DUX4 fibers; 8,443 uninduced TICDUX4 fibers; 4,079 Tam-treated WT fibers; 2,868 untreated WT fibers. Female tri, $n=9,181$ induced TICDUX4 fibers; 10,834 uninduced TIC-DUX4 fibers; 4,242 Tam-treated WT fibers; 3,773 untreated WT fibers. Male diaphragm, $n=6,917$ induced TIC-DUX4 fibers; 7,606 uninduced TIC-DUX4 fibers; 3,435 Tam-treated WT fibers; 3,362 untreated WT fibers. Female diaphragm, $n=8,246$ induced TIC-DUX4 fibers; 9,704 
uninduced TIC-DUX4 fibers; 2,266 Tam-treated WT fibers; and 3,614 untreated WT fibers.

IHC. Gene expression and subcellular localization of DUX4 protein was visualized using V5 immunofluorescence. In short, freshly cut slides were washed for 1 minute with PBS, fixed with 4\% PFA for 10 minutes, washed twice for 5 minutes with PBS and twice for 5 minutes TBST ( $0.1 \%$ tween), and incubated with V5 antibody (1:1,000; Abcam, catalog AB9116) in 10\% natural goat serum PBS (Abcam, 9116) overnight at $4^{\circ} \mathrm{C}$. Slides were then washed $3 \times$ for 5 minutes with TBST and incubated with AlexaFlor 594 goat anti-rabbit secondary antibody (Invitrogen, catalog A-11037) at 1:1,000 in PBS for 2 hours at room temperature. Finally, slides were washed $3 \times 5$ minutes with TBST and $1 \times 5$ minutes with $\mathrm{H}_{2} \mathrm{O}$ and cover slipped with Vectashield Mounting Medium containing DAPI (Vector Laboratories).

AAV1.Follistatin i.m. injections. Seven- to 8-week-old TIC-DUX4 mice received $1 \times 10^{11}$ DNase resistant particles (DRP) of AAV1 unilaterally via direct 40-50 $\mu \mathrm{l}$ i.m. injection into TA and gas muscles. Mice were treated with either AAV1.Follistatin or saline control. One week after injection, mice were induced for 8 weeks with $5 \mathrm{mg} / \mathrm{kg} 1 \times /$ week and then sacrificed 8.5 weeks after the first Tam induction.

Tam dosing. To induce Cre recombination and turn on DUX4, mice were treated with Tam via oral gavage. Tam (MilliporeSigma) was dissolved in $95 \%$ ethanol at $10 \%$ final volume and then diluted in sunflower seed oil to $15 \mathrm{mg} / \mathrm{ml}$ concentration. Tam was further diluted in sunflower oil to $0.5 \mathrm{mg} / \mathrm{ml}$ concentration. Mice were given $5 \mathrm{mg} / \mathrm{kg}$ Tam doses 1 day per week for 8 weeks.

TA and gas muscle physiology measurements. Mice were anesthetized with $137.5 \mathrm{mg} / \mathrm{kg}$ ketamine/10 mg/ $\mathrm{kg}$ xylazine. Hind limb skin was removed to expose the TA and gas muscles, and the patella. A double square knot was tied around the distal TA tendon or Achilles tendon with a 4-0 suture at the myotendinous junction, and then the tendon was cut. The exposed muscle was constantly dampened with saline. Mice were then transferred to a thermal controlled platform and maintained at $37^{\circ} \mathrm{C}$. The knee was secured to the platform with a needle through the patella tendon; the distal tendon was sutured to the level arm of the force transducer (Aurora Scientific); and the foot was secured with tape. The TA/gas muscle contractions were elicited by stimulating the sciatic nerve via bipolar platinum electrodes. Once the muscle was stabilized, the optimal length was determined by incrementally stretching the muscle until the maximum twitch force was achieved. After a 3-minute rest period, the muscle was stimulated at 50,100, 150, and $200 \mathrm{~Hz}$, allowing a 1-minute rest period between each stimulus to determine maximum tetanic force. Muscle length was measured. The mice were then euthanized, and the TA/gas muscle was removed, weighed, and frozen for analysis. Specific force was calculated by dividing the maximum tetanic force by the TA muscle cross-sectional area.

Diaphragm tetanic contraction force measurement. Diaphragm force measurements were conducted as previously described (55). In short, mice were euthanized, and the diaphragm was dissected with rib attachments and central tendon intact and placed in Krebs-Henseleit (K-H). A 2- to 4-mm-wide section of diaphragm was isolated. Using braided surgical silk (6/0, Surgical Specialties), diaphragm strips were tied at the central tendon and sutured through a portion of rib bone attached to the distal end. Each muscle was transferred to a water bath filled with oxygenated K-H solution (MilliporeSigma) that was maintained at $37^{\circ} \mathrm{C}$. The muscles were aligned horizontally and tied directly between a fixed pin and a dual-mode force transducer servomotor (305C, Aurora Scientific). Two platinum plate electrodes were positioned in the organ bath to flank the length of the muscle. The muscle was stretched to optimal length for measurement of twitch contractions and then allowed to rest for 10 minutes before initiation of the tetanic protocol. When the muscle was stabilized, the muscle was set to an optimal tension of $1 \mathrm{~g}$ and subjected to a warm-up that consisted of three $1-\mathrm{Hz}$ twitches every 30 seconds, followed by three $150-\mathrm{Hz}$ twitches every minute. The diaphragm was allowed to rest for 3 minutes. Then, the diaphragm was stimulated at $20,50,80,120,150$, and $180 \mathrm{~Hz}$ for $250 \mathrm{~ms}$ each to determine maximum tetanic force. Each stimulation was followed by a 2-minute rest period. Muscle length and weight were measured. The absolute force was normalized for muscle weight and length to calculate specific force.

Open field cage activity. An open field activity chamber was used to measure total activity as previously described (55). In short, 8-week- to 1.5 -year-old mice were analyzed using the photobeam activity system (San Diego Instruments). These studies included both induced and uninduced TIC-DUX4 mice, along with WT littermates. The grid of infrared beams measured the position and activity of each mouse in an $\mathrm{x}-\mathrm{y}-\mathrm{z}$ plane. Each mouse activity was recorded $3 \times$ per week for 1 -hour sessions in 5 min intervals. Mice were acclimated to the chamber 5 minutes before each session began. Data were exported to Excel and the $\mathrm{x}$ - and $\mathrm{y}$-plane data were added to calculate total activity. The z-plane data was used to calculate rearing activity. The average of the 3 sessions was used for each mouse. The overall average was calculated in 
GraphPad Prism software. One-way ANOVA was used to calculate $P$ values.

Statistics. All statistical analyses were performed in GraphPad Prism 5 (GraphPad Software, La Jolla, CA). Statistical tests are indicated in the figure legend for each section. One-way ANOVA with Tukey's multiple comparisons tests (Figures 1, 5, and 6); 2-way ANOVA with Tukey's multiple comparisons tests (Figures 2-4).

Study approval. All procedures were carried out in accordance with institutional policies at Nationwide Children's Hospital (IACUC no. 02605AR).

\section{Author contributions}

CRG performed TIC-DUX4 mouse characterization experiments, Follistatin injections, physiology measurements, data analysis, and experimental design and wrote the manuscript. LMW performed central nuclei and fiber diameter measurements and assisted with experimental design and data analysis. $\mathrm{KNH}$ performed physiology of TA and diaphragm muscles. JOE performed Western blot protein analysis and central nuclei and fiber diameter measurements. NYS, NKP, AMF, MAK, and DM performed central nuclei and fiber diameter measurements. AR performed 3' RACE and sequencing analysis from TICDUX4 cDNA. GAC maintained the TIC-DUX4 colony. JSD, SEGC, SMG, and JKM assisted in the development of the ROSA26-DUX4 knock-in mouse. KM provided AAV1.Follistatin virus and dosage advice for treatment experiments. LRRK contributed to the physiology measurement experiments. SQH designed the TIC-DUX4 mouse knock-in, directed experimental design and data analysis throughout the studies, and edited the manuscript. All authors contributed to data analysis.

\section{Acknowledgments}

We thank members of the Harper laboratory for assistance and support and TRINCH Transgenic Mouse Core members for assistance with ROS26-DUX4 mouse generation. This work was supported by grants from the FSH Society (grants FSHS-82015-SG3), Muscular Dystrophy Association (MDA480357; MDA218892) Wellstone Muscular Dystrophy Centers at Nationwide Children's Hospital (U54HD066409 to CRG); the National Center for Advancing Translational Sciences Award (TL1TR001069 to CRG); OSU/NCH Center for Muscle Health and Neuromuscular Disorders (82132515 to RG). During the course of this study, SQH's salary was supported by the NIH grants 1R01AR062123, 1R21NS101166, 5U54HD060848, and the NIAMS Center of Research Translation in Muscular Dystrophy Therapeutic Development Grant (1P50AR070604). During the course of this study, JE's salary was supported by the Friends of FSH Research, Chris Carrino Foundation, and the FSH Society (grants FSHD-82015-02 and FSHS-22017-03), while NS's salary was supported by the FSH Society (grants FSHS-22016-05 and FSHD-82017-01). The content is solely the responsibility of the authors and does not necessarily represent the official views of the National Center for Advancing Translational Sciences or the NIH.

Address correspondence to: Scott Q. Harper, 700 Childrens Drive, Room WA3015, Columbus, Ohio 43205, USA. Phone: 614.355.2893; Email: Scott.Harper@nationwidechildrens.org.

JD's present address is: Oregon Health and Science University, Portland, Oregon, USA.

DM's present address is: West-Ward Pharmaceuticals, Columbus, Ohio, USA.

1. Flanigan K. Facioscapulohumeral muscular dystrophy and scapuloperoneal disorders. In: Engel A, Franzini-Armstrong C, eds. Myology. New York, NY: McGraw-Hill Medical Publishing Division; 2004:1123-1133.

2. Deenen JC, et al. Population-based incidence and prevalence of facioscapulohumeral dystrophy. Neurology. 2014;83(12):1056-1059.

3. Padberg G. Facioscapulohumeral Disease. Netherlands; Leiden University: 1982.

4. Statland JM, Tawil R. Risk of functional impairment in Facioscapulohumeral muscular dystrophy. Muscle Nerve 2014;49(4):520-527

5. Statland J, Tawil R. Facioscapulohumeral muscular dystrophy. Neurol Clin. 2014;32(3):721-728.

6. Bosnakovski D, et al. An isogenetic myoblast expression screen identifies DUX4-mediated FSHD-associated molecular pathologies. EMBO J. 2008;27(20):2766-2779.

7. Dixit M, et al. DUX4, a candidate gene of facioscapulohumeral muscular dystrophy, encodes a transcriptional activator of PITX1. Proc Natl Acad Sci USA. 2007;104(46):18157-18162.

8. Gabriëls J, et al. Nucleotide sequence of the partially deleted D4Z4 locus in a patient with FSHD identifies a putative gene with in each $3.3 \mathrm{~kb}$ element. Gene. 1999;236(1):25-32. 
9. Kowaljow V, et al. The DUX4 gene at the FSHD1A locus encodes a pro-apoptotic protein. Neuromuscul Disord. 2007;17(8):611-623. 10. Lemmers RJ, et al. A unifying genetic model for facioscapulohumeral muscular dystrophy. Science. 2010;329(5999):1650-1653.

11. Richards M, Coppée F, Thomas N, Belayew A, Upadhyaya M. Facioscapulohumeral muscular dystrophy (FSHD): an enigma unravelled? Hum Genet. 2012;131(3):325-340.

12. Wijmenga C, et al. Chromosome 4q DNA rearrangements associated with facioscapulohumeral muscular dystrophy. Nat Genet. 1992;2(1):26-30.

13. de Greef JC, Frants RR, van der Maarel SM. Epigenetic mechanisms of facioscapulohumeral muscular dystrophy. Mutat Res. 2008;647(1-2):94-102.

14. van der Maarel SM, Frants RR, Padberg GW. Facioscapulohumeral muscular dystrophy. Biochim Biophys Acta. 2007;1772(2):186-194.

15. van Deutekom JC, et al. FSHD associated DNA rearrangements are due to deletions of integral copies of a $3.2 \mathrm{~kb}$ tandemly repeated unit. Hum Mol Genet. 1993;2(12):2037-2042.

16. Zeng W, et al. Specific loss of histone H3 lysine 9 trimethylation and HP1gamma/cohesin binding at D4Z4 repeats is associated with facioscapulohumeral dystrophy (FSHD). PLoS Genet. 2009;5(7):e1000559.

17. van Overveld PG, et al. Hypomethylation of D4Z4 in 4q-linked and non-4q-linked facioscapulohumeral muscular dystrophy. Nat Genet. 2003;35(4):315-317.

18. Lemmers RJ, et al. Facioscapulohumeral muscular dystrophy is uniquely associated with one of the two variants of the 4q subtelomere. Nat Genet. 2002;32(2):235-236.

19. Wuebbles RD, Long SW, Hanel ML, Jones PL. Testing the effects of FSHD candidate gene expression in vertebrate muscle development. Int J Clin Exp Pathol. 2010;3(4):386-400.

20. Mitsuhashi H, Mitsuhashi S, Lynn-Jones T, Kawahara G, Kunkel LM. Expression of DUX4 in zebrafish development recapitulates facioscapulohumeral muscular dystrophy. Hum Mol Genet. 2013;22(3):568-577.

21. Bosnakovski D, Choi SH, Strasser JM, Toso EA, Walters MA, Kyba M. High-throughput screening identifies inhibitors of DUX4-induced myoblast toxicity. Skelet Muscle. 2014;4(1):4.

22. Bosnakovski D, et al. Muscle pathology from stochastic low level DUX4 expression in an FSHD mouse model. Nat Commun. 2017;8(1):550

23. de Greef JC, et al. Smchd1 haploinsufficiency exacerbates the phenotype of a transgenic FSHD1 mouse model. Hum Mol Genet. 2018;27(4):716-731.

24. Krom YD, et al. Intrinsic epigenetic regulation of the D4Z4 macrosatellite repeat in a transgenic mouse model for FSHD. PLoS Genet. 2013;9(4):e1003415

25. Dandapat A, et al. Dominant lethal pathologies in male mice engineered to contain an X-linked DUX4 transgene. Cell Rep. 2014;8(5):1484-1496.

26. Dandapat A, et al. High Frequency Hearing Loss and Hyperactivity in DUX4 Transgenic Mice. PLoS ONE 2016;11(3):e0151467.

27. Friedrich G, Soriano P. Promoter traps in embryonic stem cells: a genetic screen to identify and mutate developmental genes in mice. Genes Dev. 1991;5(9):1513-1523.

28. McCarthy JJ, Srikuea R, Kirby TJ, Peterson CA, Esser KA. Inducible Cre transgenic mouse strain for skeletal muscle-specific gene targeting. Skelet Muscle. 2012;2(1):8.

29. Al-Zaidy SA, Sahenk Z, Rodino-Klapac LR, Kaspar B, Mendell JR. Follistatin Gene Therapy Improves Ambulation in Becker Muscular Dystrophy. J Neuromuscul Dis. 2015;2(3):185-192.

30. Haidet AM, et al. Long-term enhancement of skeletal muscle mass and strength by single gene administration of myostatin inhibitors. Proc Natl Acad Sci USA. 2008;105(11):4318-4322.

31. Kota J, et al. Follistatin gene delivery enhances muscle growth and strength in nonhuman primates. Sci Transl Med. 2009;1(6):6ra15

32. Mendell JR, et al. A phase 1/2a follistatin gene therapy trial for becker muscular dystrophy. Mol Ther. 2015;23(1):192-201

33. Rodino-Klapac LR, Haidet AM, Kota J, Handy C, Kaspar BK, Mendell JR. Inhibition of myostatin with emphasis on follistatin as a therapy for muscle disease. Muscle Nerve. 2009;39(3):283-296.

34. Rodino-Klapac LR, et al. Micro-dystrophin and follistatin co-delivery restores muscle function in aged DMD model. Hum Mol Genet. 2013;22(24):4929-4937.

35. Wallace LM, et al. DUX4, a candidate gene for facioscapulohumeral muscular dystrophy, causes p53-dependent myopathy in vivo. Ann Neurol. 2011;69(3):540-552.

36. Yao Z, et al. DUX4-induced gene expression is the major molecular signature in FSHD skeletal muscle. Hum Mol Genet. 2014;23(20):5342-5352.

37. Winokur ST, et al. Expression profiling of FSHD muscle supports a defect in specific stages of myogenic differentiation. Hum Mol Genet. 2003;12(22):2895-2907.

38. Zhang Y, et al. Human skeletal muscle xenograft as a new preclinical model for muscle disorders. Hum Mol Genet. 2014;23(12):3180-3188

39. Jones TI, Parilla M, Jones PL. Transgenic Drosophila for Investigating DUX4 and FRG1, Two Genes Associated with Facioscapulohumeral Muscular Dystrophy (FSHD). PLoS ONE. 2016;11(3):e0150938.

40. Sakellariou $P$, et al. Neuromuscular electrical stimulation promotes development in mice of mature human muscle from immortalized human myoblasts. Skelet Muscle. 2016;6:4.

41. Jagannathan S, et al. Model systems of DUX4 expression recapitulate the transcriptional profile of FSHD cells. Hum Mol Genet. 2016;25(20):4419-4431

42. Snider L, et al. Facioscapulohumeral dystrophy: incomplete suppression of a retrotransposed gene. PLoS Genet. 2010;6(10):e1001181.

43. Bosnakovski D. DUX4 in Cells and Mice. FSH Society. https://www.fshsociety.org/assets/pdf/FSHD_ASHG_IRC2008_ Philadelphia_11Nov_ProgramAndAbstracts_proof.pdf. Accessed November 6. 2018.

44. Jones T, Jones PL. A cre-inducible DUX4 transgenic mouse model for investigating facioscapulohumeral muscular dystrophy. 
PLoS ONE. 2018;13(2):e0192657.

45. Shadle SC, et al. DUX4-induced dsRNA and MYC mRNA stabilization activate apoptotic pathways in human cell models of facioscapulohumeral dystrophy. PLoS Genet. 2017;13(3):e1006658.

46. Jones TI, et al. Facioscapulohumeral muscular dystrophy family studies of DUX4 expression: evidence for disease modifiers and a quantitative model of pathogenesis. Hum Mol Genet. 2012;21(20):4419-4430.

47. Ansseau E, et al. Aberrant splicing in transgenes containing introns, exons, and V5 epitopes: lessons from developing an FSHD mouse model expressing a D4Z4 repeat with flanking genomic sequences. PLoS ONE. 2015;10(3):e0118813.

48. Wallace LM, et al. Pre-clinical Safety and Off-Target Studies to Support Translation of AAV-Mediated RNAi Therapy for FSHD. Mol Ther Methods Clin Dev. 2018;8:121-130.

49. Wallace LM, et al. RNA interference inhibits DUX4-induced muscle toxicity in vivo: implications for a targeted FSHD therapy. Mol Ther. 2012;20(7):1417-1423

50. Wagner KR, et al. A phase I/IItrial of MYO-029 in adult subjects with muscular dystrophy. Ann Neurol. 2008;63(5):561-571.

51. Campbell C, et al. Myostatin inhibitor ACE-031 treatment of ambulatory boys with Duchenne muscular dystrophy: Results of a randomized, placebo-controlled clinical trial. Muscle Nerve. 2017;55(4):458-464.

52. Langley B, Thomas M, Bishop A, Sharma M, Gilmour S, Kambadur R. Myostatin inhibits myoblast differentiation by down-regulating MyoD expression. J Biol Chem. 2002;277(51):49831-49840.

53. Lee SJ. Quadrupling muscle mass in mice by targeting TGF-beta signaling pathways. PLoS ONE. 2007;2(8):e789.

54. Lee SJ, McPherron AC. Regulation of myostatin activity and muscle growth. Proc Natl Acad Sci USA. 2001;98(16):9306-9311.

55. Pozsgai ER, Griffin DA, Heller KN, Mendell JR, Rodino-Klapac LR. Systemic AAV-Mediated $\beta$-Sarcoglycan Delivery Targeting Cardiac and Skeletal Muscle Ameliorates Histological and Functional Deficits in LGMD2E Mice. Mol Ther. 2017;25(4):855-869. 